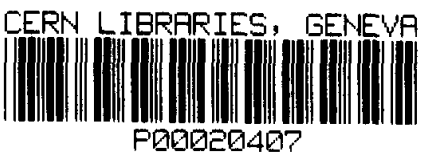

CERN-PPE/94-02

5 January 1994

89905

\title{
Invariant Mass Dependence of Particle Correlations in Hadronic Final States from the Decay of the $Z^{0}$
}

\author{
DELPHI Collaboration
}

\begin{abstract}
Two-particle correlations in invariant mass are studied separately for likesign and unlike-sign pions. Strong correlations are observed only at small masses. The correlations decrease very rapidly for like-sign pions and slowly for unlike-sign pions, manifesting different particle dynamics. The like-sign correlations exhibit a power-law behaviour as a function of mass, compatible with the previously observed intermittency effect. The predictions of the parton shower model (JETSET 7.3) deviate significantly from the data. However, good agreement is obtained with the mass correlations, both for the unlike-sign and for the like-sign pairs if the production of the $\eta^{\prime}$ and $\rho^{0}$ mesons is reduced and Bose-Einstein correlations are included in the model. The value of the Bose-Einstein correlation strength is consistent with unity for pions which are produced directly or in the decays of short-lived resonances.
\end{abstract}




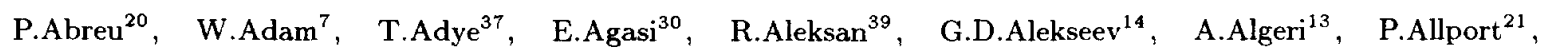
S.Almehed $^{23}, \quad$ S.J.Alvsvaag ${ }^{4}$, U.Amaldi $^{7}, \quad$ A.Andreazza ${ }^{27}$, P.Antilogus ${ }^{24}$, W-D.Apel ${ }^{15}$, R.J.Apsimon ${ }^{37}$, Y.Arnoud $^{39}$, B.Asman ${ }^{45}$, J-E.Augustin ${ }^{18}$, A.Augustinus ${ }^{30}$, P.Baillon ${ }^{7}$, P.Bambade ${ }^{18}$, F.Barao ${ }^{20}$, R.Barate ${ }^{12}$, G.Barbiellini $^{47}$, D.Y.Bardin ${ }^{14}$, G.J.Barker ${ }^{34}$, A.Baroncelli ${ }^{41}$, O.Barring ${ }^{7}$, J.A.Barrio ${ }^{25}$, W.Bartl ${ }^{50}$, M.J.Bates ${ }^{37}$, M.Battaglia ${ }^{13}$, M.Baubillier ${ }^{22}$, K-H.Becks ${ }^{52}$, M.Begalli ${ }^{36}$, P.Beilliere ${ }^{6}$, Yu.Belokopytov ${ }^{43}$, P.Beltran ${ }^{9}$, A.C.Benvenuti ${ }^{5}$, M.Berggren ${ }^{18}$, D.Bertrand ${ }^{2}$, F.Bianchi ${ }^{46}$, M.Bigi ${ }^{46}$, M.S.Bilenky ${ }^{14}$, P.Billoir ${ }^{22}$, J.Bjarne ${ }^{23}$, D.Bloch $^{8}$, J.Blocki ${ }^{51}$, S.Blyth ${ }^{34}$, V.Bocci ${ }^{38}$, P.N.Bogolubov ${ }^{14}$, T.Bolognese ${ }^{39}$, M.Bonesini ${ }^{27}$, W.Bonivento ${ }^{27}$, P.S.L.Booth ${ }^{21}$, G.Borisov ${ }^{43}$, H.Borner ${ }^{7}$, C.Bosio ${ }^{41}$, B.Bostjancic ${ }^{44}$, S.Bosworth ${ }^{34}$, O.Botner ${ }^{48}$, B.Bouquet ${ }^{18}$, C.Bourdarios $^{18}$, T.J.V.Bowcock ${ }^{21}$, M.Bozzo ${ }^{11}$, S.Braibant ${ }^{2}$, P.Branchini ${ }^{41}$, K.D.Brand ${ }^{35}$, R.A.Brenner ${ }^{7}$, H.Briand ${ }^{22}$, C.Bricman ${ }^{2}$, L.Brillault ${ }^{22}$, R.C.A.Brown ${ }^{7}$, P.Bruckman ${ }^{16}$, J-M.Brunet ${ }^{6}$, A.Budziak ${ }^{16}$, L.Bugge $^{32}$, T.Buran $^{32}$, H.Burmeister ${ }^{7}$, A.Buys ${ }^{7}$, J.A.M.A.Buytaert ${ }^{7}$, M.Caccia ${ }^{27}$, M.Calvi ${ }^{27}$, A.J.Camacho Rozas ${ }^{42}$, R.Campion ${ }^{21}$, T.Camporesi ${ }^{7}$, V.Canale ${ }^{38}$, K.Cankocak ${ }^{45}$, F.Cao ${ }^{2}$ F.Carena ${ }^{7}$ L.Carroll ${ }^{21}$, M.V.Castillo Gimenez ${ }^{49}$, A.Cattai ${ }^{7}$, F.R.Cavallo ${ }^{5}$, L.Cerrito ${ }^{38}$, V.Chabaud ${ }^{7}$, A.Chan ${ }^{1}$, M.Chapkin $^{43}$, Ph.Charpentier $^{7}$, J.Chauveau ${ }^{22}$, P.Checchia ${ }^{35}$, G.A.Chelkov ${ }^{14}$, L.Chevalier ${ }^{39}$, P.Chliapnikov ${ }^{43}$, V.Chorowicz ${ }^{22}$, J.T.M.Chrin ${ }^{49}$, V.Cindro ${ }^{44}$, P.Collins ${ }^{34}$, J.L.Contreras ${ }^{18}$, R.Contri ${ }^{11}$, E.Cortina ${ }^{49}$, G.Cosme ${ }^{18}$, F.Couchot ${ }^{18}$, H.B.Crawley ${ }^{1}$, D.Crennell ${ }^{37}$, G.Crosetti ${ }^{11}$, J.Cuevas Maestro ${ }^{33}$, S.Czellar ${ }^{13}$, E.Dahl-Jensen ${ }^{28}$, J.Dahm ${ }^{52}$, B.Dalmagne ${ }^{18}$, M.Dam ${ }^{32}$, G.Damgaard ${ }^{28}$, G.Darbo ${ }^{11}$, E.Daubie ${ }^{2}$, A.Daum ${ }^{15}$, P.D.Dauncey ${ }^{7}$, M.Davenport ${ }^{7}$, J.Davies $^{21}$, W.Da Silva ${ }^{22}$, C.Defoix ${ }^{6}$, P.Delpierre ${ }^{26}$, N.Demaria ${ }^{46}$, A.De Angelis ${ }^{7}$, H.De Boeck ${ }^{2}$, W.De Boer ${ }^{15}$, S.De Brabandere ${ }^{2}$, C.De Clercq ${ }^{2}$, M.D.M.De Fez Laso ${ }^{49}$, C.De La Vaissiere ${ }^{22}$, B.De Lotto ${ }^{47}$, A.De Min ${ }^{27}$, H.Dijkstra ${ }^{7}$, L.Di Ciaccio ${ }^{38}$, F.Djama ${ }^{8}$, J.Dolbeau ${ }^{6}$, M.Donszelmann ${ }^{7}$, K.Doroba ${ }^{51}$, M.Dracos ${ }^{8}$, J.Drees ${ }^{52}$, M.Dris $^{31}$, Y.Dufour ${ }^{7}$, F.Dupont ${ }^{12}$, D.Edsall ${ }^{1}$, L-O.Eek ${ }^{48}$, P.A.-M.Eerola ${ }^{7}$, R.Ehret ${ }^{15}$, T.Ekelof ${ }^{48}$, G.Ekspong ${ }^{45}$, A.Elliot Peisert ${ }^{7}$, M.Elsing ${ }^{52}$, J-P.Engel ${ }^{8}$, N.Ershaidat ${ }^{22}$, M.Espirito Santo ${ }^{20}$, V.Falaleev ${ }^{43}$, D.Fassouliotis ${ }^{31}$, M.Feindt ${ }^{7}$, A.Ferrer ${ }^{49}$, T.A.Filippas ${ }^{31}$, A.Firestone ${ }^{1}$, H.Foeth ${ }^{7}$, E.Fokitis ${ }^{31}$, F.Fontanelli ${ }^{11}$, K.A.J.Forbes ${ }^{21}$, J-L.Fousset ${ }^{26}$, S.Francon ${ }^{24}$, B.Franek ${ }^{37}$, P.Frenkiel ${ }^{6}$, D.C.Fries ${ }^{15}$, A.G.Frodesen ${ }^{4}$, R.Fruhwirth ${ }^{50}$, F.Fulda-Quenzer ${ }^{18}$, H.Furstenau ${ }^{15}$, J.Fuster ${ }^{7}$, D.Gamba ${ }^{46}$, M.Gandelman ${ }^{17}$, C.Garcia ${ }^{49}$, J.Garcia $^{42}$, C.Gaspar $^{7}$, U.Gasparini $^{35}$, Ph.Gavillet ${ }^{7}$, E.N.Gazis ${ }^{31}$, J-P.Gerber ${ }^{8}$, P.Giacomelli ${ }^{7}$, D.Gillespie ${ }^{7}$, R.Gokieli ${ }^{51}$, B.Golob ${ }^{44}$, V.M.Golovatyuk ${ }^{14}$, J.J.Gomez Y Cadenas ${ }^{7}$, G.Gopal ${ }^{37}$, L.Gorn ${ }^{1}$, M.Gorski ${ }^{51}$, V.Gracco ${ }^{11}$, A.Grant ${ }^{7}$, F.Grard ${ }^{2}$, E.Graziani ${ }^{41}$, G.Grosdidier ${ }^{18}$, E.Gross ${ }^{7}$, B.Grossetete ${ }^{22}$, P.Gunnarsson ${ }^{45}$, J.Guy ${ }^{37}$, U.Haedinger ${ }^{15}$, F.Hahn ${ }^{52}$, M.Hahn ${ }^{45}$, S.Hahn ${ }^{52}$, S.Haider ${ }^{30}$, Z.Hajduk ${ }^{16}$, A.Hakansson ${ }^{23}$, A.Hallgren ${ }^{48}$, K.Hamacher ${ }^{52}$, G.Hamel De Monchenault ${ }^{39}$, W.Hao ${ }^{30}$, F.J.Harris ${ }^{34}$, V.Hedberg ${ }^{23}$, T.Henkes ${ }^{7}$, R.Henriques ${ }^{20}$, J.J.Hernandez ${ }^{49}$, J.A.Hernando ${ }^{49}$, P.Herquet ${ }^{2}$, H.Herr ${ }^{7}$, T.L.Hessing ${ }^{21}$, 1.Hietanen ${ }^{13}$, C.O.Higgins ${ }^{21}$, E.Higon ${ }^{49}$, H.J.Hilke ${ }^{7}$, T.S.Hill ${ }^{1}$, S.D.Hodgson ${ }^{34}$, T.Hofmokl ${ }^{51}$, S-O.Holmgren ${ }^{45}$, P.J.Holt ${ }^{34}$, D.Holthuizen ${ }^{30}$, P.F.Honore ${ }^{6}$, M.Houlden ${ }^{21}$, J.Hrubec ${ }^{50}$, K.Huet ${ }^{2}$, K.Hultqvist ${ }^{45}$, P.Ioannou ${ }^{3}$, P-S.Iversen ${ }^{4}$, J.N.Jackson ${ }^{21}$, R.Jacobsson ${ }^{45}$, P.Jalocha ${ }^{16}$, G.Jarlskog ${ }^{23}$, P.Jarry ${ }^{39}$, B.Jean-Marie ${ }^{18}$, E.K.Johansson ${ }^{45}$, M.Jonker ${ }^{7}$, L.Jonsson ${ }^{23}$, P.Juillot ${ }^{8}$, G.Kalkanis $^{3}$, G.Kalmus ${ }^{37}$, F.Kapusta ${ }^{22}$, M.Karlsson ${ }^{45}$, E.Karvelas ${ }^{9}$, S.Katsanevas ${ }^{3}$, E.C.Katsoufis ${ }^{31}$, R.Keranen $^{7}$, B.A.Khomenko ${ }^{14}$, N.N.Khovanski ${ }^{14}$, B.King ${ }^{21}$, N.J.Kjaer ${ }^{7}$, H.Klein ${ }^{7}$, A.Klovning ${ }^{4}$, P.Kluit ${ }^{30}$, A.Koch-Mehrin $^{52}$, J.H.Koehne ${ }^{15}$, B.Koene ${ }^{30}$, P.Kokkinias ${ }^{9}$, M.Koratzinos ${ }^{32}$, A.V.Korytov ${ }^{14}$, V.Kostioukhine ${ }^{43}$, C.Kourkoumelis $^{3}$, O.Kouznetsov ${ }^{14}$, P.H.Kramer ${ }^{52}$, M.Krammer ${ }^{50}$, C.Kreuter ${ }^{15}$, J.Krolikowski ${ }^{51}$, I.Kronkvist ${ }^{23}$, W.Kucewicz ${ }^{16}$, K.Kulka ${ }^{48}$, K.Kurvinen ${ }^{13}$, C.Lacasta ${ }^{49}$, C.Lambropoulos ${ }^{9}$, J.W.Lamsa ${ }^{1}, \quad$ L.Lanceri $^{47}$, P.Langefeld ${ }^{52}$, V.Lapin ${ }^{43}$, I.Last ${ }^{21}$, J-P.Laugier ${ }^{39}$, R.Lauhakangas ${ }^{13}$, G.Leder ${ }^{50}$, F.Ledroit ${ }^{12}$, R.Leitner ${ }^{29}$, Y.Lemoigne $^{39}$, J.Lemonne ${ }^{2}$, G.Lenzen ${ }^{52}$, V.Lepeltier ${ }^{18}$, J.M.Levy ${ }^{8}$, E.Lieb ${ }^{52}$, D.Liko ${ }^{50}$, J.Lindgren ${ }^{13}$, R.Lindner $^{52}$, A.Lipniacka ${ }^{18}$, I.Lippi ${ }^{35}$, B.Loerstad ${ }^{23}$, M.Lokajicek ${ }^{10}$, J.G.Loken ${ }^{34}$, A.Lopez-Fernandez $^{7}$, M.A.Lopez Aguera ${ }^{42}$, M.Los ${ }^{30}$, D.Loukas ${ }^{9}$, J.J.Lozano ${ }^{49}$, P.Lutz ${ }^{6}$, L.Lyons ${ }^{34}$, G.Maehlum ${ }^{32}$, J.Maillard ${ }^{6}$, A.Maio $^{20}$, A.Maltezos ${ }^{9}$, F.Mandl ${ }^{50}$, J.Marco ${ }^{42}$, M.Margoni ${ }^{35}$, J-C.Marin ${ }^{7}$, C.Mariotti ${ }^{41}$, A.Markou ${ }^{9}$, T.Maron $^{52}$, S.Marti ${ }^{49}$, C.Martinez-Rivero ${ }^{42}$, F.Martinez-Vidal ${ }^{49}$, F.Matorras ${ }^{42}$, C.Matteuzzi ${ }^{27}$, G.Matthiae ${ }^{38}$, M.Mazzucato $^{35}$, M.Mc Cubbin ${ }^{21}$, R.Mc Kay ${ }^{1}$, R.Mc Nulty ${ }^{21}$, J.Medbo ${ }^{48}$ ， C.Meroni2 ${ }^{27}$, W.T.Meyer ${ }^{1}$, M.Michelotto $^{35}$, L.Mirabito ${ }^{24}$, W.A.Mitaroff ${ }^{50}$, G.V.Mitselmakher ${ }^{14}$, U.Mjoernmark ${ }^{23}$, T.Moa ${ }^{45}$, R.Moeller ${ }^{28}$, K.Moenig $^{7}$, M.R.Monge ${ }^{11}$, P.Morettini ${ }^{11}$, H.Mueller ${ }^{15}$, W.J.Murray ${ }^{37}$, B.Muryn ${ }^{16}$, G.Myatt ${ }^{34}$, F.Naraghi ${ }^{12}$, F.L.Navarria ${ }^{5}$, P.Negri ${ }^{27}$, S.Nemecek ${ }^{10}$, W.Neumann ${ }^{52}$, N.Neumeister ${ }^{50}$, R.Nicolaidou ${ }^{3}$, B.S.Nielsen ${ }^{28}$, V.Nikolaenko ${ }^{43}$, P.E.S.Nilsen ${ }^{4}$, P.Niss ${ }^{45}$, A.Nomerotski ${ }^{35}$, V.Obraztsov ${ }^{43}$, A.G.Olshevski ${ }^{14}$, R.Orava $^{13}$, A.Ostankov $^{43}$, K.Osterberg ${ }^{13}$, A.Ouraou ${ }^{39}$, P.Paganini ${ }^{18}$, M.Paganoni ${ }^{27}$, R.Pain ${ }^{22}$, H.Palka ${ }^{16}$, Th.D.Papadopoulou ${ }^{31}$, L.Pape ${ }^{7}$, F.Parodi ${ }^{11}$, A.Passeri ${ }^{41}$, M.Pegoraro ${ }^{35}$, J.Pennanen ${ }^{13}$, L.Peralta ${ }^{20}$, V.Perevozchikov ${ }^{43}$, H.Pernegger ${ }^{50}$, M.Pernicka ${ }^{50}$, A.Perrotta ${ }^{5}$, C.Petridou ${ }^{47}$, A.Petrolini ${ }^{11}$, G.Piana ${ }^{11}$, F.Pierre $^{39}$, M.Pimenta ${ }^{20}$, S.Plaszczynski ${ }^{18}$, O.Podobrin ${ }^{15}$, M.E.Pol ${ }^{17}$, G.Polok ${ }^{16}$, P.Poropat ${ }^{47}$, V.Pozdniakov ${ }^{14}$, M.Prest $^{47}$, P.Privitera ${ }^{38}$, A.Pullia ${ }^{27}$, D.Radojicic ${ }^{34}$, S.Ragazzi ${ }^{27}$, H.Rahmani ${ }^{31}$, J.Rames ${ }^{10}$, P.N.Ratof ${ }^{19}$, A.L.Read ${ }^{32}$, M.Reale ${ }^{52}$, P.Rebecchi ${ }^{18}$, N.G.Redaelli ${ }^{27}$, M.Regler ${ }^{50}$, D.Reid ${ }^{7}$, P.B.Renton ${ }^{34}$, L.K.Resvanis ${ }^{3}$, F.Richard ${ }^{18}$, J.Richardson ${ }^{21}$, J.Ridky ${ }^{10}$, G.Rinaudo ${ }^{46}$, A.Romero ${ }^{46}$, I.Roncagliolo ${ }^{11}$, P.Ronchese ${ }^{35}$, C.Ronnqvist $^{13}$, E.I.Rosenberg ${ }^{1}$, E.Rosso ${ }^{7}$, P.Roudeau ${ }^{18}$, T.Rovelli ${ }^{5}$, W.Ruckstuhl ${ }^{30}$, V.Ruhlmann-Kleider ${ }^{39}$, 
A.Ruiz ${ }^{42}$, K.Rybicki ${ }^{16}$, H.Saarikko ${ }^{13}$, Y.Sacquin ${ }^{39}$, G.Sajot $^{12}$, J.Salt ${ }^{49}$, J.Sanchez ${ }^{25}$, M.Sannino ${ }^{11,40}$, S.Schael ${ }^{7}$, H.Schneider ${ }^{15}$, M.A.E.Schyns ${ }^{52}$, G.Sciolla ${ }^{46}$, F.Scuri ${ }^{47}$, A.M.Segar ${ }^{34}$, A.Seitz ${ }^{15}$, R.Sekulin ${ }^{37}$, M.Sessa ${ }^{47}$, R.Seufert ${ }^{15}$, R.C.Shellard ${ }^{36}$, I.Siccama ${ }^{30}$, P.Siegrist ${ }^{39}$, S.Simonetti ${ }^{11}$, F.Simonetto ${ }^{35}$, A.N.Sisakian ${ }^{14}$, G.Skjevling $^{32}$, G.Smadja ${ }^{39,24}$, O.Smirnova ${ }^{14}$, G.R.Smith ${ }^{37}$, R.Sosnowski ${ }^{51}$, D.Souza-Santos ${ }^{36}$, T.Spassov ${ }^{20}$ E.Spiriti ${ }^{41}$, S.Squarcia ${ }^{11}$, H.Staeck ${ }^{52}$, C.Stanescu ${ }^{41}$, S.Stapnes ${ }^{32}$, G.Stavropoulos ${ }^{9}$, F.Stichelbaut ${ }^{2}$, A.Stocchi ${ }^{18}$ J.Strauss $^{50}$, J.Straver ${ }^{7}$, R.Strub ${ }^{8}$, B.Stugu ${ }^{4}$, M.Szczekowski ${ }^{7}$, M.Szeptycka ${ }^{51}$, P.Szymanski ${ }^{51}$, T.Tabarelli ${ }^{27}$, O.Tchikilev ${ }^{43}$, G.E.Theodosiou ${ }^{9}$, A.Tilquin ${ }^{26}$, J.Timmermans ${ }^{30}$, V.G.Timofeev ${ }^{14}$, L.G.Tkatchev ${ }^{14}$, T.Todorov ${ }^{8}$, D.Z.Toet ${ }^{30}$, O.Toker ${ }^{13}$, A.Tomaradze ${ }^{2}$, B.Tome ${ }^{20}$, E.Torassa ${ }^{46}$, L.Tortora ${ }^{41}$, D.Treille ${ }^{7}$, W.Trischuk ${ }^{7}$, G.Tristram ${ }^{6}$, C.Troncon ${ }^{27}$, A.Tsirou ${ }^{7}$, E.N.Tsyganov ${ }^{14}$, M.Turala $^{16}$, M-L.Turluer ${ }^{39}$, T.Tuuva ${ }^{13}$, I.A.Tyapkin ${ }^{22}$ M.Tyndel $^{37}$, S.Tzamarias ${ }^{21}$, B.Ueberschaer ${ }^{52}$, S.Ueberschaer ${ }^{52}$, O.Ullaland ${ }^{7}$, V.Uvarov ${ }^{43}$, G.Valenti ${ }^{5}$, E.Vallazza ${ }^{7}$, J.A.Valls Ferrer ${ }^{4}$, C.Vander Velde ${ }^{2}$, G.W.Van Apeldoorn ${ }^{30}$, P.Van Dam ${ }^{30}$, M.Van Der Heijden ${ }^{30}$, W.K.Van Doninck ${ }^{2}$, J.Van Eldik ${ }^{30}$, P.Vaz ${ }^{7}$, G.Vegni ${ }^{27}$, L.Ventura ${ }^{35}$, W.Venus ${ }^{37}$, F.Verbeure ${ }^{2}$, M.Verlato ${ }^{35}$, L.S.Vertogradov ${ }^{14}$, D.Vilanova ${ }^{39}$, P.Vincent ${ }^{24}$, L.Vitale ${ }^{47}$, E.Vlasov ${ }^{43}$, A.S.Vodopyanov ${ }^{14}$, M.Vollmer ${ }^{52}$, M.Voutilainen ${ }^{13}$, V.Vrba ${ }^{41}$, H.Wahlen $^{52}$, C.Walck ${ }^{45}$, F.Waldner ${ }^{47}$, A.Wehr ${ }^{52}$, M.Weierstall ${ }^{52}$, P.Weilhammer ${ }^{7}$, A.M.Wetherell $^{7}$, J.H.Wickens ${ }^{2}$, M.Wielers ${ }^{15}$, G.R.Wilkinson ${ }^{34}$, W.S.C.Williams ${ }^{34}$, M.Winter ${ }^{8}$, G.Wormser ${ }^{18}$, K.Woschnagg ${ }^{48}$, A.Zaitsev ${ }^{43}$, A.Zalewska ${ }^{16}$, D.Zavrtanik ${ }^{44}$, E.Zevgolatakos ${ }^{9}$, N.I.Zimin ${ }^{14}$, M.Zito $^{39}$, D.Zontar ${ }^{44}$, R.Zuberi ${ }^{34}$, G.Zumerle ${ }^{35}$, J.Zuniga ${ }^{49}$

\footnotetext{
${ }^{1}$ Ames Laboratory and Department of Physics, Iowa State University, Ames IA 50011, USA

${ }^{2}$ Physics Department, Univ. Instelling Antwerpen, Universiteitsplein 1, B-2610 Wilrijk, Belgium and IIHE, ULB-VUB, Pleinlaan 2, B-1050 Brussels, Belgium

and Faculté des Sciences, Univ. de l'Etat Mons, Av. Maistriau 19, B-7000 Mons, Belgium

${ }^{3}$ Physics Laboratory, University of Athens, Solonos Str. 104, GR-10680 Athens, Greece

${ }^{4}$ Department of Physics, University of Bergen, Allégaten 55, N-5007 Bergen, Norway

${ }^{5}$ Dipartimento di Fisica, Università di Bologna and INFN, Via Irnerio 46, I-40126 Bologna, Italy

${ }^{6}$ Collège de France, Lab. de Physique Corpusculaire, IN2P3-CNRS, F-75231 Paris Cedex 05, France

${ }^{\top} \mathrm{CERN}, \mathrm{CH}-1211$ Geneva 23, Switzerland

${ }^{8}$ Centre de Recherche Nucléaire, IN2P3 - CNRS/ULP - BP20, F-67037 Strasbourg Cedex, France

${ }^{9}$ Institute of Nuclear Physics, N.C.S.R. Demokritos, P.O. Box 60228, GR-15310 Athens, Greece

${ }^{10} \mathrm{FZU}$, Inst. of Physics of the C.A.S. High Energy Physics Division, Na Slovance 2, CS-180 40, Praha 8, Czechoslovakia

${ }^{11}$ Dipartimento di Fisica, Università di Genova and INFN, Via Dodecaneso 33, I-16146 Genova, Italy

${ }^{12}$ Institut des Sciences Nucléaires, IN2P3-CNRS, Université de Grenoble 1, F-38026 Grenoble, France

${ }^{13}$ Research Institute for High Energy Physics, SEFT, P.O. Box 9, FIN-00014 University of Helsinki, Finland

14 Joint Institute for Nuclear Research, Dubna, Head Post Office, P.O. Box 79, 101000 Moscow, Russian Federation

${ }^{15}$ Institut für Experimentelle Kernphysik, Universität Karlsruhe, Postfach 6980, D-76128 Karisruhe, Germany

${ }^{16}$ High Energy Physics Laboratory, Institute of Nuclear Physics, Ul. Kawiory 26a, PL-30055 Krakow 30 , Poland

${ }^{17}$ Centro Brasileiro de Pesquisas Físicas, rua Xavier Sigaud 150, RJ-22290 Rio de Janeiro, Brazil

${ }^{18}$ Université de Paris-Sud, Lab. de l'Accélérateur Linéaire, IN2P3-CNRS, Bat 200, F-91405 Orsay, France

${ }^{19}$ School of Physics and Materials, University of Lancaster, GB-Lancaster LA1 4YB, UK

${ }^{20}$ LIP, IST, FCUL - Av. Elias Garcia, 14-1 ${ }^{\circ}$, P-1000 Lisboa Codex, Portugal

${ }^{21}$ Department of Physics, University of Liverpool, P.O. Box 147, GB-Liverpool L69 3BX, UK

${ }^{22}$ LPNHE, IN2P3-CNRS, Universités Paris VI et VII, Tour 33 (RdC), 4 place Jussieu, F-75252 Paris Cedex 05, France ${ }^{23}$ Department of Physics, University of Lund, Sölvegatan 14, S-22363 Lund, Sweden

${ }^{24}$ Université Claude Bernard de Lyon, IPNL, IN2P3-CNRS, F-69622 Villeurbanne Cedex, France

${ }^{25}$ Universidad Complutense, Avda. Complutense s/n, E-28040 Madrid, Spain

${ }^{26}$ Univ. d'Aix - Marseille II - CPP, IN2P3-CNRS, F-13288 Marseille Cedex 09, France

${ }^{27}$ Dipartimento di Fisica, Università di Milano and INFN, Via Celoria 16, I-20133 Milan, Italy

${ }^{28}$ Niels Bohr Institute, Blegdamsvej 17 , DK-2100 Copenhagen 0, Denmark

${ }^{29} \mathrm{NC}$, Nuclear Centre of MFF, Charles University, Areal MFF, V Holesovickach 2, CS-180 00, Praha 8, Czechoslovakia

${ }^{30}$ NIKHEF-H, Postbus 41882, NL-1009 DB Amsterdam, The Netherlands

${ }^{1}$ National Technical University, Physics Department, Zografou Campus, GR-15773 Athens, Greece

${ }^{32}$ Physics Department, University of Oslo, Blindern, N-1000 Oslo 3, Norway

${ }^{33}$ Dpto. Fisica, Univ. Oviedo, C/P.Jimenez Casas, S/N-33006 Oviedo, Spain

${ }^{34}$ Department of Physics, University of Oxford, Keble Road, Oxford OX1 3RH, UK

${ }^{35}$ Dipartimento di Fisica, Università di Padova and INFN, Via Marzolo 8, I-35131 Padua, Italy

${ }^{36}$ Depto. de Fisica, Pontificia Univ. Católica, C.P. 38071 RJ-22453 Rio de Janeiro, Brazil

${ }^{37}$ Rutherford Appleton Laboratory, Chilton, GB - Didcot OX11 OQX, UK

${ }^{38}$ Dipartimento di Fisica, Università di Roma II and INFN, Tor Vergata, I-00173 Rome, Italy

${ }^{39}$ Centre d'Etude de Saclay, DSM/DAPNIA, F-91191 Gif-sur-Yvette Cedex, France

${ }^{40}$ Dipartimento di Fisica-Università di Salerno, I-84100 Salerno, Italy

${ }^{41}$ Istituto Superiore di Sanità, Ist. Naz. di Fisica Nucl. (INFN), Viale Regina Elena 299, I-00161 Rome, Italy

42 C.E.A.F.M., C.S.I.C. - Univ. Cantabria, Avda. los Castros, S/N-39006 Santander, Spain

43 Inst. for High Energy Physics, Serpukov P.O. Box 35, Protvino, (Moscow Region), Russian Federation

${ }^{44} \mathrm{~J}$. Stefan Institute and Department of Physics, University of Ljubljana, Jamova 39, SI-61000 Ljubljana, Slovenia

${ }^{45}$ Fysikum, Stockholm University, Box 6730, S-113 85 Stockholm, Sweden

46 Dipartimento di Fisica Sperimentale, Universita di Torino and INFN, Via P. Giuria 1, I-10125 Turin, Italy

${ }^{47}$ Dipartimento di Fisica, Università di Trieste and INFN, Via A. Valerio 2, I-34127 Trieste, Italy

and Istituto di Fisica, Università di Udine, I-33100 Udine, Italy

${ }^{48}$ Department of Radiation Sciences, University of Uppsala, P.O. Box 535, S-751 21 Uppsala, Sweden

${ }^{49}$ IFIC, Valencia-CSIC, and D.F.A.M.N., U. de Valencia, Avda. Dr. Moliner 50, E-46100 Burjassot (Valencia), Spain

${ }^{50}$ Institut für Hochenergiephysik, Österr. Akad. d. Wissensch., Nikolsdorfergasse 18, A-1050 Vienna, Austria

${ }^{51}$ Inst. Nuclear Studies and University of Warsaw, Ul. Hoza 69, PL-00681 Warsaw, Poland

${ }^{52}$ Fachbereich Physik, University of Wuppertal, Postfach 100 127, D-5600 Wuppertal 1, Germany
} 


\section{Introduction}

Particle correlations have been studied for many years in terms of a variety of kinematical variables. Recently, considerable effort has been devoted to the study of fluctuation phenomena in multiparticle production processes, both from a theoretical and an experimental point of view [1]. These studies concentrated on the search for intermittency, i.e. the occurrence of fluctuations due to scale-invariant dynamics. The search is made by studying normalized factorial moments $F_{q}$ of order $q$ of the multiplicity distributions of charged particles as a function of the size $\delta$ of phase space cells [2]. The $F_{q}$ are defined by:

$$
F_{q}(\delta)=\frac{<n(n-1) \ldots(n-q+1)>}{<n>^{q}} .
$$

Intermittency in particle production is defined as a power-law behaviour of $F_{q}$ as a function of $\delta$ :

$$
F_{q}(\delta) \sim \delta^{-\phi_{q}}
$$

where the intermittency index $\phi_{q}$ is a positive constant. The first studies were performed in one dimensional phase-space, in the kinematical variable rapidity $y$ or pseudo-rapidity $\eta$. Later it was realized that the effect is smeared out by the projection from momentum space onto one dimension [3] and therefore two and three dimensional analyses were undertaken in terms of three variables: rapidity $y$, azimuthal angle $\varphi$ and $\ln p_{T}$, the transverse momentum, or combinations of these. It was soon realized that a study of factorial moments amounts to a study of correlations [4].

The most recent development in the study of intermittency is the use of the "Correlation Integral" method [5], which indicated that the invariant quantity $Q^{2}=-\left(q_{1}-q_{2}\right)^{2}$ (where $q_{1}$ and $q_{2}$ are the four-momenta of the particles) is a more suitable variable than the traditional ones because it combines the features of a three-dimensional analysis with the large statistics of the one-dimensional projection [6]. For two-pion correlations, the invariant mass of the studied pair is related to $Q^{2}$ by:

$$
Q^{2}=M^{2}-4 m_{\pi}^{2}
$$

Therefore, the invariant mass $M$ of the particle pair is an appropriate variable to study two-particle correlations. Although the information obtained from $Q^{2}$ distributions is the same as the one obtained from $M$ distributions, it is advantagous to use the latter variable for the following reasons. Intermittency in terms of the factorial cumulant or Mueller moment [7] $K_{2}=F_{2}-1$ has the form:

$$
K_{2}\left(Q^{2}\right) \sim\left(\frac{1}{Q^{2}}\right)^{\beta}
$$

which leads to singularities for $Q^{2} \rightarrow 0$. The use of $M^{2}$ or $M$ instead of $Q^{2}$ avoids such singularity and gives a finite correlation strength at the two pion mass threshold. It is also a more familiar variable with a clear physical meaning, directly related to resonance signals and their reflections. In this paper the effects of correlations (intermittency) are studied by analyzing two-pion correlations separately for unlike-sign and for likesign pairs. The study of the latter, influenced by Bose-Einstein correlations, is another motivation for the present study. Indeed, very different values of the source size $(r)$ and correlation strength $(\lambda)$ are obtained in LEP experiments [8-10] using the same Gaussian parametrization for the Bose-Einstein correlation but two different reference samples for the uncorrelated background. 


\section{Analysis}

The two-particle correlation function is defined as the inclusive coincidence rate per collision, minus the rate expected if the hadrons are uncorrelated:

$$
C_{2}\left(q_{a}, q_{b}\right) \equiv \rho_{2}^{a b}\left(q_{a}, q_{b}\right)-\rho_{1}^{a}\left(q_{a}\right) \rho_{1}^{b}\left(q_{b}\right),
$$

where $q_{a}$ and $q_{b}$ are the four-momenta of the particles $a$ and $b$. The two-particle inclusive density is:

$$
\rho_{2}^{a b}\left(q_{a}, q_{b}\right)=\frac{E_{a} E_{b}}{N_{\mathrm{ev}}} \frac{d^{6} n}{d^{3} p_{a} d^{3} p_{b}}
$$

with $n$ the number of pairs and $N_{\text {ev }}$ the number of events. The single-particle density is:

$$
\rho_{1}(q)=\frac{E}{N_{\mathrm{ev}}} \frac{d^{3} n}{d^{3} p}
$$

with $n$ the number of particles. The normalizations are:

$$
\begin{aligned}
\iint \rho_{2}^{a b}\left(q_{a}, q_{b}\right) \frac{d^{3} p_{a}}{E_{a}} \frac{d^{3} p_{b}}{E_{b}} & =<n_{a}\left(n_{b}-\delta_{a b}\right)> \\
\int \rho_{1}^{a, b}(q) \frac{d^{3} p}{E_{a, b}} & =<n_{a, b}>
\end{aligned}
$$

with $\delta_{a b}=1$ if particles $(a)$ and $(b)$ are identical and zero otherwise.

Integrating (5) over all variables except the effective mass $M$ of the pairs gives

$$
C_{2}(M)=\rho_{2}(M)-\rho_{1} \otimes \rho_{1}(M)
$$

where $\rho_{2}(M)$ is the two-particle invariant mass distribution divided by the number of events and with normalization (8). The second term in (10) describes the uncorrelated background and is given by:

$$
\rho_{1}^{a} \otimes \rho_{1}^{b}(M)=\int \frac{d^{3} p_{a}}{E_{a}} \frac{d^{3} p_{b}}{E_{b}} \delta\left(\sqrt{\left(q_{a}+q_{b}\right)^{2}}-M\right) \rho_{1}^{a}\left(q_{a}\right) \rho_{1}^{b}\left(q_{b}\right)
$$

with normalization:

$$
\int \rho_{1}^{a} \otimes \rho_{1}^{b}(M) d M=<n_{a}><n_{b}>.
$$

The uncorrelated background (11) was calculated using four-vectors ${ }^{\dagger}$ of particles randomly selected from different events. The quantity $\rho_{1} \otimes \rho_{1}(M)$ was then calculated in the same way as for real events, and normalized to the value given by (12) in the kinematical region considered.

The ratio:

$$
R(M)=\frac{\rho_{2}(M)}{\rho_{1} \otimes \rho_{1}(M)}
$$

is a direct measure of correlations. If calculated for all charge combinations, $R(M)$ is equal to $F_{2}(\mathrm{M})$, the normalized factorial moment of order two, and to $K_{2}(M)+1$. In this paper the search for correlation effects is carried out by studying the ratio $R(M)$ for like-sign and unlike-sign combinations.

\footnotetext{
${ }^{\dagger}$ Momentum components of the particles are calculated with respect to the thrust axis
} 


\section{Event and track selection}

The present study is based on the sample of hadronic events collected with the DELPHI detector during 1992 , at a centre-of-mass energy of $\sqrt{s}=91.28 \mathrm{GeV}$. The detector has been described in ref. [11]. In this analysis all charged particles are assumed to be pions and only those which satisfy the following requirements are used:

- polar angle $\theta$ with respect to the beam axis between $25^{\circ}$ and $155^{\circ}$;

- momentum larger than $0.2 \mathrm{GeV} / \mathrm{c}$ and smaller than $50 \mathrm{GeV} / \mathrm{c}$;

- measured track length in the TPC, the main tracking chamber of DELPHI, larger than $50 \mathrm{~cm}$;

- measured impact parameter with respect to the event vertex within $5 \mathrm{~cm}$ in the transverse plane and $10 \mathrm{~cm}$ along the beam direction.

Hadronic events were selected by requiring that:

- there are at least 5 charged particles in the event;

- the total energy of charged particles exceeds $3 \mathrm{GeV}$, in each of the two hemispheres with respect to the beam axis;

- the total energy of all charged particles is larger than $15 \mathrm{GeV}$;

- the total momentum imbalance of all charged particles is less than $30 \mathrm{GeV} / \mathrm{c}$;

- the polar angle of the thrust axis satisfies $\left|\cos \theta_{\text {th }}\right|<0.75$.

A sample of 490,440 events satisfied these requirements. The contamination from events due to beam-gas scattering, $\gamma \gamma$ interactions and $\tau^{+} \tau^{-}$events is estimated to be less than $0.3 \%$ of the selected events [12]. To ensure that the analysis is restricted to charged particles originating from the primary vertex, invariant masses of particle pairs were calculated for particles with impact parameter relative to the event vertex, within $0.1 \mathrm{~cm}$ in the transverse plane and $1 \mathrm{~cm}$ along the beam direction. The strict cuts on both transverse and longitudinal impact parameters remove most tracks from $K_{S}^{0}$ and $\Lambda$ decays but keep the particles from charm and bottom decays. To exclude overlapping tracks with potential problems which might not be correctly reproduced in the simulation, the following procedure was used: all pairs of tracks were considered and, if the opening angle was less than $2^{\circ}$, the shorter track was removed from the event. The stability of the results was checked by varying the cut on opening angle up to $3^{\circ}$.

The Monte Carlo simulation program DELSIM [13] was used to correct the data for geometrical acceptance, kinematical cuts, resolution and particle interactions with the detector material. A sample of $Z^{0}$ events was generated with JETSET 7.3PS [14] and all particles were followed through the detector. The simulated events were processed through the same reconstruction chain as real data. A sample of 300,000 events passed the track and event selection criteria used for the data sample. Correction factors were calculated per bin of effective mass as the ratio of the generated to the accepted number of pairs.

In the following, all comparisons between the data and the JETSET predictions are presented with corrected distributions. However, it was checked that the same features are present at the level of the uncorrected distributions.

\section{Results}

The correlation functions $C_{2}(M)$ and $R(M)$ were first studied in ref. [15] using a small sample of $p p$ interactions. Recently, it was shown in a large statistics $\pi^{+} p / K^{+} p$ 
experiment [16] that relation (4) holds for the variable $M$, separately for like-sign and unlike-sign pion pairs.

In the absence of kinematical and dynamical correlations $R(M)$ would be strictly one, and $C_{2}(M)$ zero. Decay products of a resonance will affect the relevant mass correlations around the resonance mass. Combinations of two particles out of three or more body decays of a resonance, should also lead to sizable correlations. For example, $\pi^{+} \pi^{-}$ combinations from $\eta$ and $\eta^{\prime}$ should contribute at small invariant masses.

\subsection{Correlations in invariant mass}

Fig. 1 shows $\rho_{2}(M)$, the inclusive two-particle density, $\rho_{1} \otimes \rho_{1}(M)$, the uncorrelated background, and the correlation $C_{2}(M)$ for unlike-sign and for like-sign pairs, for the corrected DELPHI data. The mass dependent correction factors are shown in Fig. 2a, $2 \mathrm{~b}$ and $2 \mathrm{c}$ respectively for $\rho_{2}(M)$, the background and $R(M)$. The shape of the $\rho_{1} \otimes \rho_{1}(M)$ function is identical for the like-sign and the unlike-sign combinations because they are derived from the single-particle distributions which are the same for positive and negative particles. From Fig. 1a it is seen that the +- correlations are significant for small effective masses $\left(\leq 1 \mathrm{GeV} / \mathrm{c}^{2}\right)$, with additional contributions around the $\rho^{0}(770)$ and $f_{0}(975)$ masses. The like-sign correlation decreases more rapidly with increasing mass than the unlike-sign one and becomes negative for $M \geq 0.65 \mathrm{GeV} / \mathrm{c}^{2}$. The same observations are valid if only particles with $|y|<2$ are considered (not shown), where $y$ is the rapidity of the particle along the thrust axis. The latter cut is used in most intermittency analyses [17-19].

Fig. 2 shows the correction factors for $\rho_{2}(M), \rho_{1} \otimes \rho_{1}(M)$ and $R(M)$. The former are of the order of 2.4 for pairs, corresponding to about 1.6 for single particles, essentially due to the strict cuts on impact parameter. Fig. $2 \mathrm{~b}$ indicates that, as expected, the correction factor for the uncorrelated background is the same for like-sign and for unlike-sign pairs at all mass values. The correction factor for the two-particle density $\rho_{2}$ is about $6 \%$ larger for like-sign than for unlike-sign pairs. Both correction factors for $\rho_{2}(M)$ and for $\rho_{1} \otimes \rho_{1}(M)$ exhibit a very sharp rise towards the two-pion mass threshold, due to the cut on $2^{\circ}$ opening angle of the pair. Therefore, in this analysis the region $M<0.34$ $\mathrm{GeV} / \mathrm{c}^{2}$, corresponding to $Q^{2}<0.04 \mathrm{GeV}^{2}$, will not be used. It is important to avoid the very small mass region for a study of Bose-Einstein effects (see Sect. 4.4), because this is precisely the region where reflections from resonances decaying into three or more particles contribute (particularly $\eta^{\prime}$ and $D^{*}$ ), and where Coulomb effects are important.

The correction factors for $R(M)$ (Fig. 2c) are equal to unity within about $5 \%$ and are smooth as a function of mass, except for the +- combination at $M \simeq 0.5 \mathrm{GeV} / \mathrm{c}$, where a small influence of $K_{S}^{0}$ decay is seen. It can also be remarked that, contrary to the correction factors for $\rho_{2}(M), \rho_{1} \otimes \rho_{1}(M)$ or $C_{2}(M)$, the correction factors for $R(M)$ practically do not depend on the momentum of the pairs.

Fig. 3 shows the ratio $R(M)$ (Eq. 13) for full phase space (Fig. 3a) and with a rapidity cut $|y|<2$ (Fig. 3b). Several features are visible in these figures:

- strong correlations are present at small masses;

- the value of $R(M)$ for unlike-sign combinations is significantly larger than for likesign pairs;

- the unlike-sign combinations display clear signals of the $\rho^{0}(770)$ and the $f_{0}(975)$ mesons; 
- for the sample of particles with $|y|<2$ (Fig. $3 \mathrm{~b}$ ), where the kinematical correlations from energy-momentum conservation are less influential, both ratios are close to one at masses above $1.8 \mathrm{GeV} / \mathrm{c}^{2}$.

\subsection{Comparison with JETSET}

The data are compared with the predictions of JETSET 7.3PS, with parameters tuned to the DELPHI results [20]. This model which does not include Bose-Einstein Correlations generally shows good agreement with $e^{+} e^{-}$data on inclusive distributions and on global event variables. Fig. 4 shows the ratio of the data to the JETSET prediction for $\rho_{2}(M)$ (unlike-sign combinations in Fig. 4a, like-sign ones in Fig. 4b) and for the uncorrelated background $\rho_{1} \otimes \rho_{1}(M)$ (Fig. 4c). Systematic differences between JETSET and the data are present in all three cases, i.e. both for the two-particle invariant mass distributions and for the uncorrelated background, with JETSET predicting too steep a decrease at larger masses. Concentrating on the small mass region where the correlations are strong, Fig. 5 shows the ratio $R(M)$ for the data and JETSET. In this ratio systematic differences at high mass values largely cancel. Nevertheless, strong disagreements are observed.

Several comments can be made for unlike-sign pairs (Fig. 5a):

- JETSET considerably underestimates $\mathrm{R}(M)$ in the mass interval from 0.4 to 0.6 $\mathrm{GeV} / \mathrm{c}^{2}$;

- The discrepancy around the $f_{0}$-mass is due to the absence of $f_{0}$ production in the version of JETSET 7.3 used [21];

- $R(M)$ in the model is slightly but systematically too large at $M>1 \mathrm{GeV} / \mathrm{c}^{2}$;

- A very steep decline of $R(M)$ from threshold to $\mathrm{M}=0.4 \mathrm{GeV} / \mathrm{c}^{2}$ is observed in JETSET, much steeper than in the data; this steep decline was traced back to the contribution of $\pi^{+} \pi^{-}$pairs from $\eta$ and $\eta^{\prime}$ decays. ALEPH has shown [22] that $\eta^{\prime}$ production is strongly overestimated in JETSET.

- A strong $\rho^{0}$ signal is observed in the model, a weaker one in the data. In Fig. 6, the ratio $R(M)$ is shown for particle pairs with a cut at $x_{\pi \pi}=0.1$, where $x_{\pi \pi}=$ $2|p| / E_{\mathrm{cm}}$ and $p$ is the momentum of the $\pi \pi$ system. The agreement between the data and the model is quite good for $x_{\pi \pi}>0.1$ (Fig. 6a), where the experimental value of the $\rho^{0}$ cross section [21] also agrees with the JETSET prediction.

No direct measurement of the $\rho^{0}$ production cross section is available at LEP energies in the kinematical region $x_{\pi \pi}<0.05$ and, therefore, the production cross section in JETSET cannot be checked. Still, Fig. $6 \mathrm{~b}$ suggests that the $\rho^{0}$ production is too abundant in the model at small $x_{\pi \pi}$ values. ${ }^{\ddagger}$ An analogous problem with $\rho^{0}$ meson production was observed in hadron-hadron interactions [23]. As seen from Fig. 6, the strong disagreement in Fig. 5a for $M=0.4 \div 0.6 \mathrm{GeV} / \mathrm{c}^{2}$ is also due to unlike-sign pairs with $x_{\pi \pi}<0.1$. In JETSET this $x_{\pi \pi}$-region contains about $85 \%$ of all $\pi^{+} \pi^{-}$pairs from $\eta^{\prime}$ decay and $65 \%$ of all $\rho^{0}$ decays. As a step towards a more realistic description of the production of $\eta^{\prime}$ and $\rho^{0}$, a sample of JETSET events was generated where $75 \%$ of the $\eta^{\prime}$ and $40 \%$ of the $\rho^{0}$ meson decays were prohibited and these fractions of the resonances taken as stable particles. The first value is based on a measurement of the $\eta^{\prime}$ production rate [22] and the second one was adjusted to give better agreement with the data. A reasonable satisfactory agreement with the data is obtained ${ }^{\S}$ (see dashed line in Fig. 6b). Most of the $\pi^{+} \pi^{-}$pairs from $\omega(783)$ decays

\footnotetext{
${ }^{\ddagger}$ A too high $\eta^{\prime}$ production rate also generates some extra $\rho^{0}$ 's at small $x_{\pi \pi}$ values, via the decay $\eta^{\prime} \rightarrow \rho^{0} \gamma$ with $30 \%$ branching ratio.

$\S$ In this version of JETSET Bose-Einstein correlations were also included for direct pions (see sect. 4.4).
} 
contribute in the mass region of 0.4 to $0.6 \mathrm{GeV} / \mathrm{c}$. Increasing the $\omega$ production rate in JETSET by $15-30 \%$ leads to an improved agreement with the data (not shown) but the sensitivity of the $R_{+-}(M)$ distribution to the $\omega$ production rate is too low to allow a conclusion.

Fig. 5b shows $R(M)$ for like-sign pairs, both for the data and the model. The experimental values of $R(M)$ are considerably higher than the JETSET predictions for $M$ smaller than $0.6 \mathrm{GeV} / \mathrm{c}^{2}$ and are lower for larger $M$. This is usually attributed to the Bose-Einstein interference. However, it is striking that JETSET also predicts a strong rise of $R(M)$ towards threshold, although no Bose-Einstein interference was present in the model. This is due to the presence of multijet and $b \bar{b}$ events [9]. The influence of multijet events is the strongest cause of the rise at small masses. Additional correlations are generated at low masses because, in the denominator of (13), combinations of tracks from different jets lead to larger masses, thereby yielding a relative depletion of $\rho_{1} \otimes \rho_{1}(M)$ at low masses and thus an increase of $R(M)$. It was verified that in JETSET $R_{l . s .}(M)$, for like-sign combinations, varies by less than $7 \%$ around an average value of 0.95 (not shown) over the mass interval up to $1.6 \mathrm{GeV} / \mathrm{c}^{2}$ if two-jet events are selected by the LUCLUS algorithm and $b \bar{b}$ events are removed.

\subsection{Intermittency in invariant mass}

Since there are no prominent resonances which decay into two like-sign charged particles, ${ }^{\top}$ the JETSET prediction of $R_{\text {l.s. }}(M)$ takes into account both the kinematical correlations and "methodological" correlations, i.e. the ones originating from the mixing of different jet multiplicities and $b \bar{b}$ events. Contrary to the case of unlike-sign combinations, $R_{l . s .}(M)$ does not change significantly in JETSET if the influence of $\eta^{\prime}$ and $\rho^{0}$ decays is reduced.

The ratio:

$$
R^{\prime}(M)=\frac{R^{\text {data }}(M)}{R_{l . s .}^{\mathrm{MC}}(M)}
$$

is a measurement of dynamical correlations. The quantity $R^{\prime}$ for like-sign and unlike-sign pairs is shown in Fig. 7a for all combinations and in Fig. 7b for particles with $|y|<2$. From this figure it can be seen that:

- most of the correlations are confined to the small mass region $M<1.8 \mathrm{GeV} / \mathrm{c}^{2}$;

- the cut $|y|<2$ hardly influences the results at all, indicating that kinematical correlations are indeed removed;

- the $\rho^{0}$ is more prominent than in Fig. 3;

- in the small mass region, the like-sign ratio decreases much faster than the unlikesign one.

Below the $\rho^{0}$ mass region, the ratio $R^{\prime}$ is fitted to the form

$$
R^{\prime}(M)=A+B\left(\frac{1}{M^{2}}\right)^{\beta}
$$

and the results of the fit with $A$ fixed to one, are given in Table 1 . This is equivalent to a fit of the factorial cumulant or Mueller moment [7] to the form:

$$
K_{2}=B\left(\frac{1}{M^{2}}\right)^{\beta}
$$

\footnotetext{
Except for the $\eta^{\prime}$ which decays 4.8 times less often in like-sign pairs than into unlike-sign ones, and the $\Delta^{++}$and $D^{*}$ which do not change the $R(M)$ distributions significantly in the mass region studied.
} 
As shown in Fig. 8, the quality of the fits is satisfactory. The values of $\beta$ for like-sign and unlike-sign pairs are very different: the slope for unlike-sign pairs is close to zero, the one for like-sign pairs is 1.5 units. A reasonable fit to the form (15) with free parameter $A$ is also obtained for the like-sign combinations in the mass interval $0.34 \div 1.0 \mathrm{GeV} / \mathrm{c}^{2}$ (last line of Table 1 and full line in Fig. 9). This analysis shows that a power-law behaviour of the factorial moments or cumulants is also observed in terms of the effective mass of the pairs. Furthermore, the mass dependence of the factorial cumulants of all charge combinations is dominated by the contributions of like-sign combinations and the intermittency slope is considerably stronger in effective mass than in rapidity [17], which is used in most intermittency studies.

\subsection{Bose-Einstein correlations}

Recently, several authors considered the possibility that Bose-Einstein (BE) correlations could be responsible for the observed intermittency effects [24] but no quantitative proof was given. In the present analysis, JETSET 7.3 PS was used without BE correlations. Previously [10], the DELPHI collaboration studied BE correlations using two different reference samples: the sample of unlike pair combinations yielding a correlation strength $\lambda=0.45 \pm 0.02$ and radius of the source $r=0.82 \pm 0.03 \mathrm{fm}$ and a sample of mixed tracks yielding the values $\lambda=0.35 \pm 0.04$ and $r=0.42 \pm 0.04 \mathrm{fm}$, in fits to the form:

$$
R^{\prime}=N(1+\delta Q)\left[1+\lambda \exp \left(-r^{2} Q^{2}\right)\right]
$$

where $N$ is a normalization factor and the term $(1+\delta Q)$ takes into account possible long range correlations. Similar results were obtained by ALEPH [9]. These results were obtained using corrections for Coulomb interactions and for non $-\pi-\pi$ pairs. The ratio $R^{\prime}(M)$ (Eq. 14) for like-sign pairs is the same quantity which was studied by DELPHI [10] with the mixing technique, but calculated as a function of $Q$, using another mixing method: two-jet events were selected and the momenta of particles from one jet were reflected through the origin, to be combined with particles from the second jet. The same Bose-Einstein parameters within errors as in [10] were obtained with the mixing method used in this paperll, without any selection on two-jet events nor cuts on momenta.

Experimentally, the $\lambda$ and $r$ parameters are measured for all pion pairs including pions from decays of long-lived particles and, consequently, the value of $\lambda$ is the mean value of the correlation strength for all pions. To compare with the data, JETSET events were generated with $\mathrm{BE}$ correlations included in the Gaussian parametrization for all pions and with both sets of parameter values: one obtained by using unlike-sign pairs and the one using a mixed track reference sample. The results, shown in Fig. 10, where the data are the same as in Fig. 5b for like-sign combinations, clearly prove that JETSET with $\mathrm{BE}$ correlations and the parameters derived in $[10]$ with the mixed track reference sample gives a good representation of the data (Fig. 10b), whereas the parameters obtained with the unlike-sign combinations (Fig. 10a) do not. Indeed, the decay products of the $\eta$ and $\eta$ ' mesons strongly influence the values of $\lambda$ and $r$ when these parameters are estimated by using the unlike-sign combinations as a reference sample (see also Figs. 5a and 6b).

It is known $[9,25]$ that pions are effectively uncorrelated within the experimental resolution if they are produced from the decays of long-lived particles. To investigate the

\footnotetext{
$\|$ The measured values of $\lambda$ and $r$ for all pions in the present analysis are $\lambda=0.33 \pm 0.02$ and $r=0.42 \pm 0.02 \mathrm{fm}$. These values were obtained using the correction for non- $\pi-\pi$ pairs but not corrected for Coulomb interactions. The correction with the Gamow factor amounts to less than $2 \%$ change in the considered mass region. The values measured without any correction are $\lambda=0.25 \pm 0.02$ and $r=0.44 \pm 0.01 \mathrm{fm}$, also in good agreement with the corresponding uncorrected values obtained in ref. [10] using the mixing technique
} 
expected influence of long-lived states on $\lambda$, a separate analysis was undertaken [25], using flavour-tagged data samples enriched in $b$ - and $u d s$-events respectively. These two samples, which differ in their contents of long-lived heavy-quark states, should in principle have different $\lambda$ values as a result of their different amounts of non-interfering pairs. A difference in the $\lambda$ value of 2.2 standard deviations between the two samples was observed, $\lambda$ being smaller for $b$-events than for the $u d s$-events. This gives experimental support to the hypothesis that long-lived heavy-quark states influence the correlation strength. In the JETSET model, the fraction of direct like-sign pion pairs** in the sample of all like-sign charge particle combinations, can be well parametrized in the low-mass region $\left(M=0.34 \div 1.20 \mathrm{GeV} / \mathrm{c}^{2}\right)$ by the function:

$$
f(Q)=0.17+0.26 Q-0.12 Q^{2} .
$$

In the above equation, only pairs are considered where both particles are direct pions or both pions are decay products from the same weakly decaying particle. The fraction of the latter ones is less than $1 \%$ in the considered mass region. One can correct for the presence of non-direct pion-pion pairs by fitting the ratio $R^{\prime}$ for like-sign pions (Fig. 7a) with a slightly modified form of (17):

$$
R^{\prime}=N(1+\delta Q)\left[1+\lambda f(Q) \exp \left(-Q^{2} r^{2}\right)\right] .
$$

The data and the result of the fit are shown in Fig. 11a. The fitted parameter values are:

$$
\lambda=1.06 \pm 0.05, \quad r=0.49 \pm 0.01 \mathrm{fm}
$$

with $\chi^{2} / \mathrm{DF}=84 / 82$.

Using these fitted values of $\lambda$ and $r$, the individual entries of the $R^{\prime}(M)$ distribution can be corrected for the finite purity of direct pion pairs by weighting each entry by a factor:

$$
\frac{1+\lambda \exp \left(-Q^{2} r^{2}\right)}{1+\lambda f(Q) \exp \left(-Q^{2} r^{2}\right)}
$$

The results are shown in Fig. $11 \mathrm{~b}$, as well as the functional form (17) with parameters obtained from the fit to function (19).

The main source of systematics for the $\lambda$ and $r$ parameters is the uncertainty on the iraction (18) of direct pion pairs, calculated by means of JETSET. To estimate the systematic errors on the parameters, this fraction was calculated by applying to the model various modifications which are relevant for this fraction:

- A systematic relative error of $\pm 10 \%$ was assigned to the fraction of pairs from decays of charm and bottom particles which are long-lived. This number was estimated using the average charge multiplicity of bottom particle decays as measured [26]. The corresponding uncertainties on the $\lambda$ and $r$ parameters are $\Delta \lambda= \pm 0.10$ and $\Delta r= \pm 0.04 \mathrm{fm}$;

- The production rates of the $\eta^{\prime}$ and $\rho^{0}$ were reduced by $75 \%$ and $40 \%$, respectively, as discussed in Sect. 4.2, and the $\omega$ production rate increased by $30 \%$. The maximal variations of $\lambda$ and $r$ were -0.12 and -0.02 , respectively, and, consequently, the uncertainties were estimated as $\Delta \lambda= \pm 0.12, \Delta r= \pm 0.02$ with respect to (20).

- The width of the Gaussian parametrization of transverse momentum distributions for primary hadrons was varied between the default value of $0.350 \mathrm{GeV} / \mathrm{c}$ and the DELPHI tuned value of $0.395 \mathrm{GeV} / \mathrm{c}$ [20], yielding uncertainties of \pm 0.04 and $\pm 0.01 \mathrm{fm}$, respectively, for $\lambda$ and $r$.

*Here and in the following, direct pions are taken to be pions which are either produced promptly or decay pions of short-lived resonances. All particles with lifetimes longer than the $K^{*}(890)$ are considered to be long-lived [14] 
- The suppression of the $s$-quark pair production was varied between 0.28 and 0.32 , the range having been determined in [27] from the $K^{0}$ differential cross section. The uncertainties are $\Delta \lambda= \pm 0.02$ and $\Delta r= \pm 0.01$.

All contributions were added in quadrature and the final values of the parameters $\lambda$ and $r$ for direct pions are:

$$
\lambda=1.06 \pm 0.05 \pm 0.16, \quad r=0.49 \pm 0.01 \pm 0.05 \mathrm{fm}
$$

where the first quoted error is statistical and the second systematic. The value of $r$ is close to the value $r=0.42 \pm 0.04 \mathrm{fm}$, measured for all pions with the mixing technique [10], whereas the $\lambda$ value found is compatible with unity, indicating maximal interference strength.

As a verification, JETSET events were generated where BE correlations were included after decays of short-lived resonances, but before decays of long-lived ones and with $\lambda=1, r=0.50 \mathrm{fm}$. Good agreement with the data on like-sign combinations is found (Fig. 12). Including BE correlations in JETSET not only changes the distribution of like-sign correlations but also of the unlike-sign ones as is shown in Fig. 13. The model with BE correlations included (full line in Fig. 13) leads to a better agreement with the data than JETSET without BE correlations (dashed line), particularly in the $\rho^{0}(770)$ mass region. The mass of the $\rho^{0}(770)$, measured from the $\pi^{+} \pi^{-}$invariant mass spectrum in this experiment [21], is five standard deviations below the Particle Data Group value [28]. This can be explained in part by the distortion of the Breit-Wigner shape of the $\rho^{0}(770)$ by $\mathrm{BE}$ correlations [29]. On the other hand, it also shows that decay pions from the $\rho^{0}$ and other short-lived resonances play an important role in the BE effect for like-sign combinations in the mass region considered in this analysis ${ }^{\dagger \dagger}$. Moreover, JETSET calculations show that the fraction of like-sign pion pairs where both pions are directly produced without being decay product of a resonance, is only about $2 \%$ in the investigated mass region. Consequently, most of the like-sign two pion correlation is due to interference of pions in pairs where at least one of the pions is the decay product of a resonance.

\section{Summary}

New results on two-pion correlations in invariant mass are obtained from a sample of about 500,000 hadronic events from DELPHI, mainly using the ratio of two-particle density to the uncorrelated production according to the single-particle density (Eq. 13).

The standard Monte Carlo program JETSET7.3 PS for the generation of hadronic $Z^{0}$ decays fails to represent the data both for like-sign and unlike-sign particle correlations.

The production of $\eta^{\prime}$ and $\rho^{0}$ is too large in JETSET and is responsible for the disagreement in unlike-sign correlations. If, in JETSET, the production rate of the $\eta^{\prime}$ is reduced according to the results of $\mathrm{ALEPH}$, and the $\rho^{0}$ production rate is tuned to the DELPHI data, reasonable agreement is obtained for unlike-sign correlations.

The Bose-Einstein effect is the main source of like-sign correlations. The maximal interference strength is needed for direct pions to explain the data. If the Bose-Einstein effect is included in JETSET, good agreement is found with the data on like-sign correlations. Correlations for like-sign combinations are responsible for most of the intermittency effect and these are dominated by Bose-Einstein correlations.

${ }^{1+}$ Less than $10 \%$ of the $\rho^{\circ}(770)$ decay products in JETSET contribute to the mass region below $0.34 \mathrm{GeV} / \mathrm{c}$ 


\section{Acknowledgements}

We are greatly indebted to our technical collaborators and to the funding agencies for their support in building and operating the DELPHI detector, and to the members of the CERN-SL Division for the excellent performance of the LEP collider. We warmly thank E.A. De Wolf, who initiated this kind of analysis in another experiment, for many fruitful discussions and $T$. Sjöstrand for useful discussions on Bose-Einstein correlations in JETSET. 


\section{References}

[1] For recent reviews, see e.g. Proc. of the Ringberg Workshop "Fluctuations and Fractal Structure", Eds. R. Hwa, W. Ochs, N. Schmitz, World Scientific, Singapore, 1992; E.A. De Wolf, I.M. Dremin, W.K. Kittel, "Scaling laws for density correlations and fluctuations in Multiparticle Dynamics", Nijmegen preprint HEN-362 (1993), Brussels preprint IIHE-ULB-VUB-93-01

[2] A. Białas, R. Peschanski: Nucl. Phys. B273 (1986) 703; B308 (1988) 857

[3] A. Białas, J. Seixas: Phys. Lett. B250 (1990) 161

[4] E.A. De Wolf, Acta Phys. Pol. , B21 (1990) 611

[5] P. Lipa et al.: Phys. Lett. B285 (1992) 300

P. Lipa et al.: Phys. Lett. B301 (1993) 298

[6] NA22 Collaboration, N. Agababyan et al., Zeit. Phys. C59 (1993) 405

[7] A.H. Mueller, Phys. Rev. D4 (1971) 150

[8] OPAL Collaboration, P. Acton et al., Phys. Lett. B267 (1991) 143.

[9] ALEPH Collaboration, D. Decamp et al., Zeit. Phys. C54 (1992) 75

[10] DELPHI Collaboration, P. Abreu et al., Phys. Lett. B286 (1992) 201

11] DELPHI Collaboration, P. Aarnio et al., Nucl. Instr. Methods A303 (1991) 233

[12] DELPHI Collaboration, P. Abreu et al., Zeit. Phys. C54 (1992) 55

[13] DELSIM User Manual, DELPHI 87-96 PROG-99, Geneva

DELSIM Reference Manual, DELPHI 87-97 PROG-100, Geneva

[14] T. Sjöstrand, Comput. Phys. Comm. 27 (1982) 243; 28 (1983) 229;

T. Sjöstrand, M. Bengtsson, Comput. Phys. Comm. 43 (1987) 367

[15] E.L. Berger, R. Singer, G.H. Thomas, T. Kafka: Phys. Rev. D15 (1977) 206

[16] E.A. De Wolf, Proc. XXII Int. Symp. on Multiparticle Dynamics, Santiago de Compostela, July 1992, Ed. C. Pajares, World Scientific, Singapore (1993). p. 263

[17] DELPHI Collaboration, P. Abreu et al., Phys. Lett. B247 (1990) 137

[18] OPAL Collaboration, M.Z. Akrawy et al., Phys. Lett. B262 (1991) 351

[19] NA22 Collaboration, I.V. Ajinenko et al., Phys. Lett. B222 (1989) 306

[20] W. De Boer et al., IEKP-KA/91-07, Karlsruhe, june 1991

[21] DELPHI Collaboration, P. Abreu et al., Phys. Lett. B298 (1993) 236

[22] ALEPH Collaboration, D. Buskelic et al., Phys. Lett. B292 (1992) 210

[23] NA22 Collaboration, I.V. Agababyan et al., Zeit. Phys. C41 (1989) 539

[24] F. Mandl, B. Buschbeck, Proc. XXIIth Int. Symp. on Multiparticle Dynamics, Santiago de Compostela, July 1992, Ed. C. Pajares, World Scientific, Singapore (1993), p. 561

K. Kadija, P. Seyboth, Phys. Lett. B287 (1992) 363

A. Białas, Acta Phys. Pol. B23 (1992) 561

[25] DELPHI Collaboration, K. Kulka, Influence on the Bose-Einstein Effect by Decaying Long-Lived States, DELPHI 93-119 PHYS-337

[26] OPAL Collaboration, P. Acton et al., Studies of charged particle multiplicity in $b$ quark events, CERN PPE/93-174

[27] DELPHI Collaboration, P. Abreu et al., Phys. Lett. 275B (1992) 231

[28] Particle Data Group, K. Hikasa et al., Phys. Rev. D45 (1992) 1

[29] G.D. Lafferty, Zeit. Phys. C60 (1993) 659 
Table 1. Fits of $R^{\prime}(M)$ to the form (15)

\begin{tabular}{|c|c|c|c|c|c|c|}
\hline Pairs & Mass (GeV) & Selection & A & B & $\beta$ & $\chi^{2} / \mathrm{NDF}$ \\
\hline+- & $0.34-0.55$ & & 1 (fixed) & $0.31 \pm 0.02$ & $0.05 \pm 0.03$ & $19 / 19$ \\
\pm \pm & $0.34-0.55$ & & 1 (fixed) & $0.0051 \pm 0.0008$ & $1.54 \pm 0.09$ & $23 / 19$ \\
+- & $0.34-0.55$ & $|y|<2$ & 1 (fixed) & $0.30 \pm 0.02$ & $0.10 \pm 0.03$ & $15 / 19$ \\
\pm \pm & $0.34-0.55$ & $|y|<2$ & 1 (fixed) & $0.0060 \pm 0.0010$ & $1.53 \pm 0.09$ & $34 / 19$ \\
\hline \pm \pm & $0.34-1.0$ & & $0.886 \pm 0.007$ & $0.058 \pm 0.010$ & $0.68 \pm 0.06$ & $81 / 63$ \\
\hline
\end{tabular}

\section{Figure Captions}

1. Two-particle density $\rho_{2}(M)$, uncorrelated background $\rho_{1} \otimes \rho_{1}(M)$ and the correlation $C_{2}(M)$ as a function of effective mass for +- and for \pm \pm pairs, corrected for acceptance.

2. Correction factors used for a) the two-particle densities, b) the uncorrelated backgrounds and $\mathbf{c}$ ) the ratios $R(M)$.

3. The ratio $R(M)$ for a) all like-sign and unlike-sign combinations, b) particles with $|y|<2$.

4. Ratio of the data to the JETSET prediction for $\rho_{2}(M)$ a) for unlike-sign and $\mathbf{b}$ ) like-sign combinations and c) for the uncorrelated background $\rho_{1} \otimes \rho_{1}(M)$.

5 . The ratio $R(M)$ for the data compared to the JETSET 7.3 prediction with parameters tuned to the DELPHI data, for a) unlike-sign and b) like-sign combinations. The data points are the same as in Fig. $3 \mathrm{a}$.

6. The ratio $R^{+-}(M)$ for pairs with a) $x_{\pi \pi}>0.1$ and b) $x_{\pi \pi}<0.1$ The full line is the JETSET prediction with parameters tuned to the DELPHI data. The dashed line is JETSET with Bose-Einstein (BE) correlations included for direct pions with $\lambda=1$. and $r=0.50 \mathrm{fm}$, and with $75 \%$ of the $\eta^{\prime}$ and $40 \%$ of the $\rho^{0}$ decays prohibited (RJ).

7. The ratio $R^{\prime}(M)$ (Eq. 14) for a) all like-sign and unlike-sign combinations and $\mathrm{b}$ ) particles with $|y|<2$.

8. The factorial cumulant $K_{2}(M)$ for unlike-sign and for like-sign combinations with power-law fits superimposed for a) all pairs and b) particles with $|y|<2$.

9. The ratio $R^{\prime}(M)$ for like-sign combinations with fit to the form (15) superimposed. The data points are the same as in Fig. $7 \mathrm{a}$.

10. The ratio $R(M)$ for like-sign pairs compared to predictions of JETSET 7.3 with $\mathrm{BE}$ correlations and parameter values a) $\lambda=0.45, r=0.82 \mathrm{fm}$ and $\mathbf{b}) \lambda=0.35$, $r=0.42 \mathrm{fm}$. BE correlations in JETSET are included for all like-sign pions. The data are the same as in Fig. $5 \mathrm{~b}$.

11. a) The ratio $R^{\prime}(M)$ for like-sign combinations (data points are the same as in Fig. $7 \mathrm{a})$ and fit to the form (19) superimposed. b) The ratio $R^{\prime}(M)$ for direct pion pairs. The function (17) is superimposed with parameters obtained by the fit with (19).

12. The ratio $R(M)$ for like-sign combinations, compared to the prediction of the JETSET model with BE correlations included for direct pions, with parameters $\lambda=1$., $r=0.50 \mathrm{fm}$. The data are the same as in Fig. 5b.

13. The ratio $R(M)$ for unlike-sign combinations in the kinematical region $x_{\pi \pi}<0.1$ (the data is the same as in Fig. 6b). The dashed line is the JETSET prediction without, the full line with $\mathrm{BE}$ correlations included. In both versions of the model, $\eta^{\prime}$ and $\rho^{0}$ production were reduced as discussed in Sect. 4.2. 
DELPHI

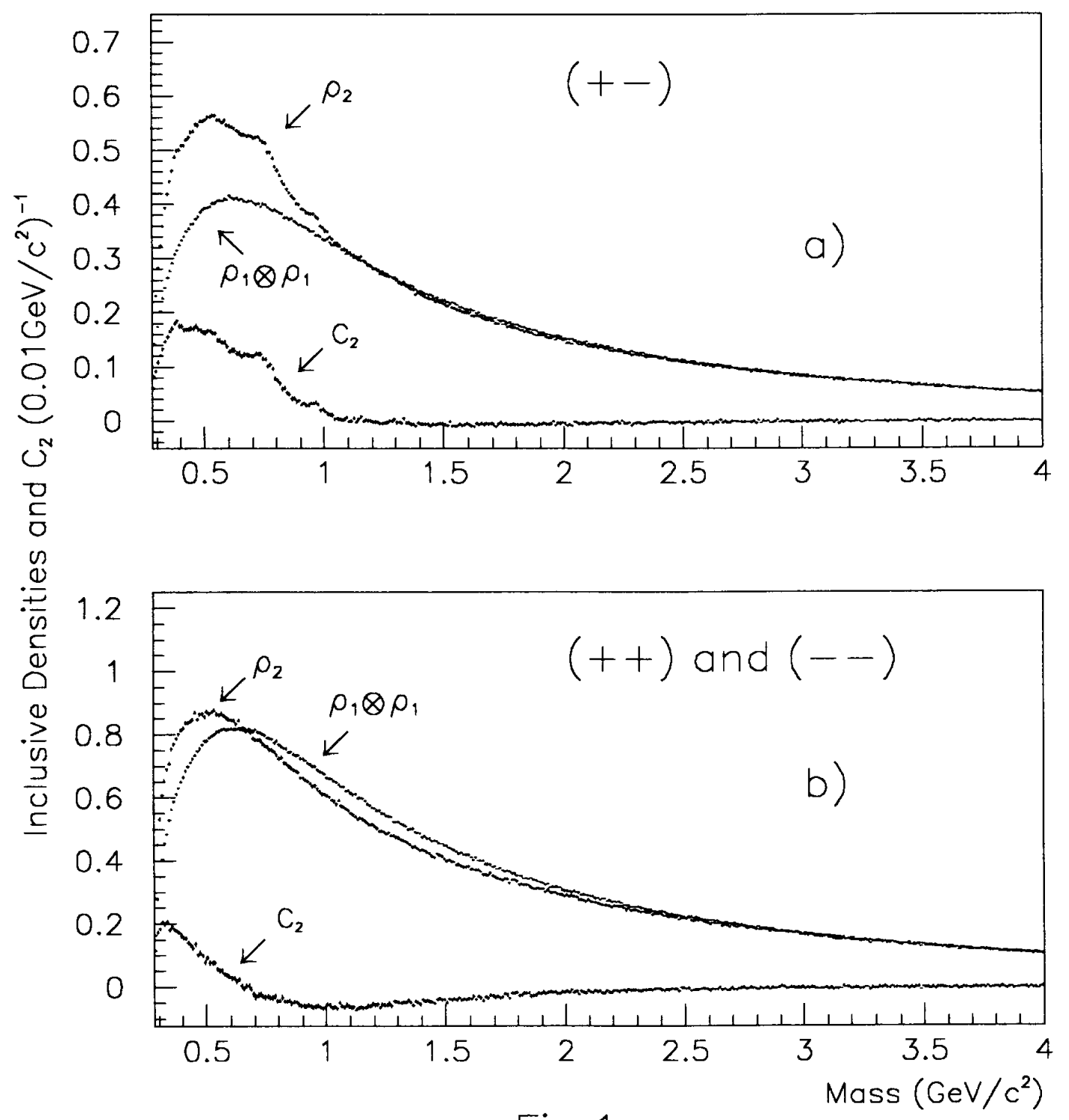

Fig.1 

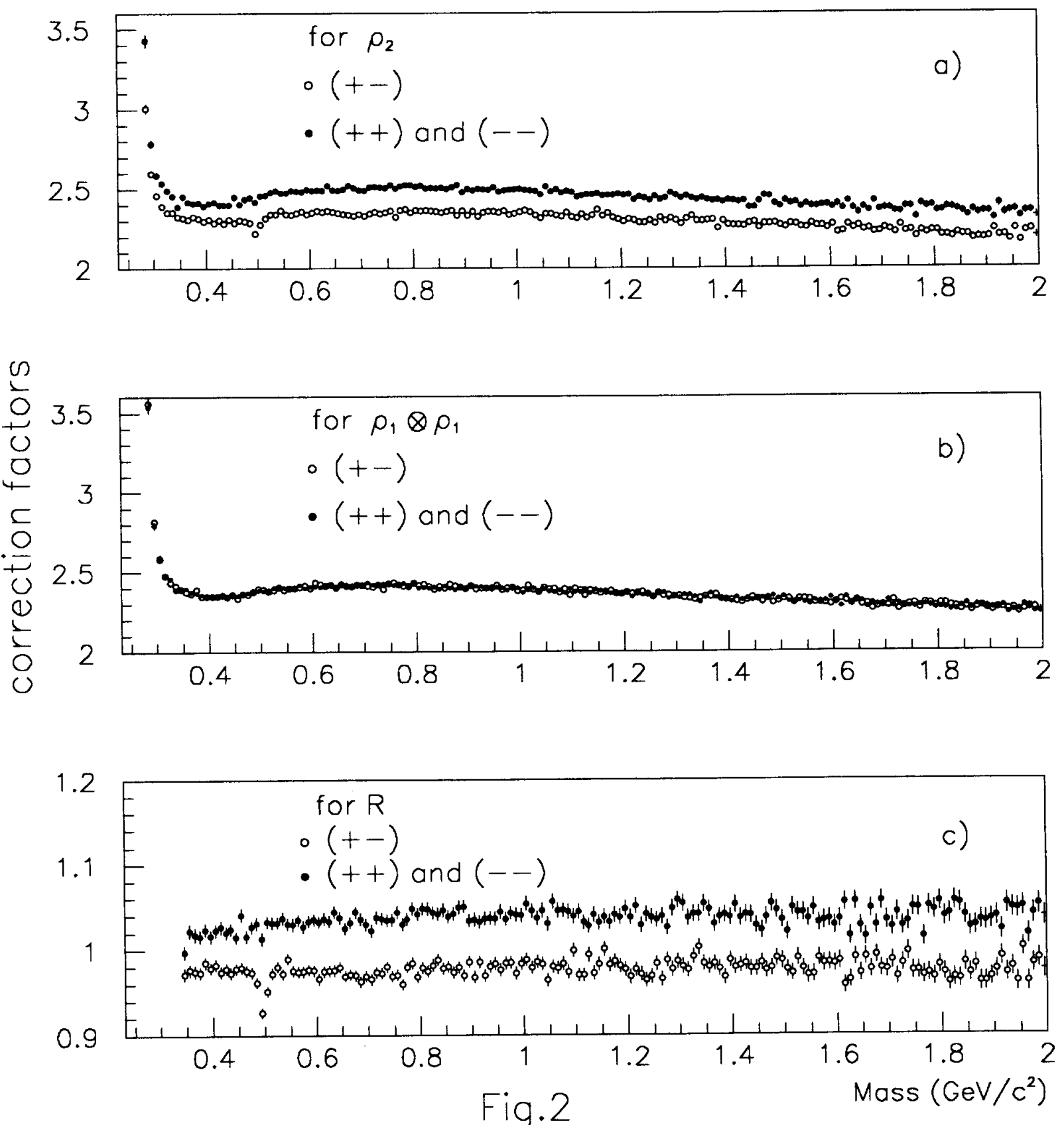


\section{DELPHI}

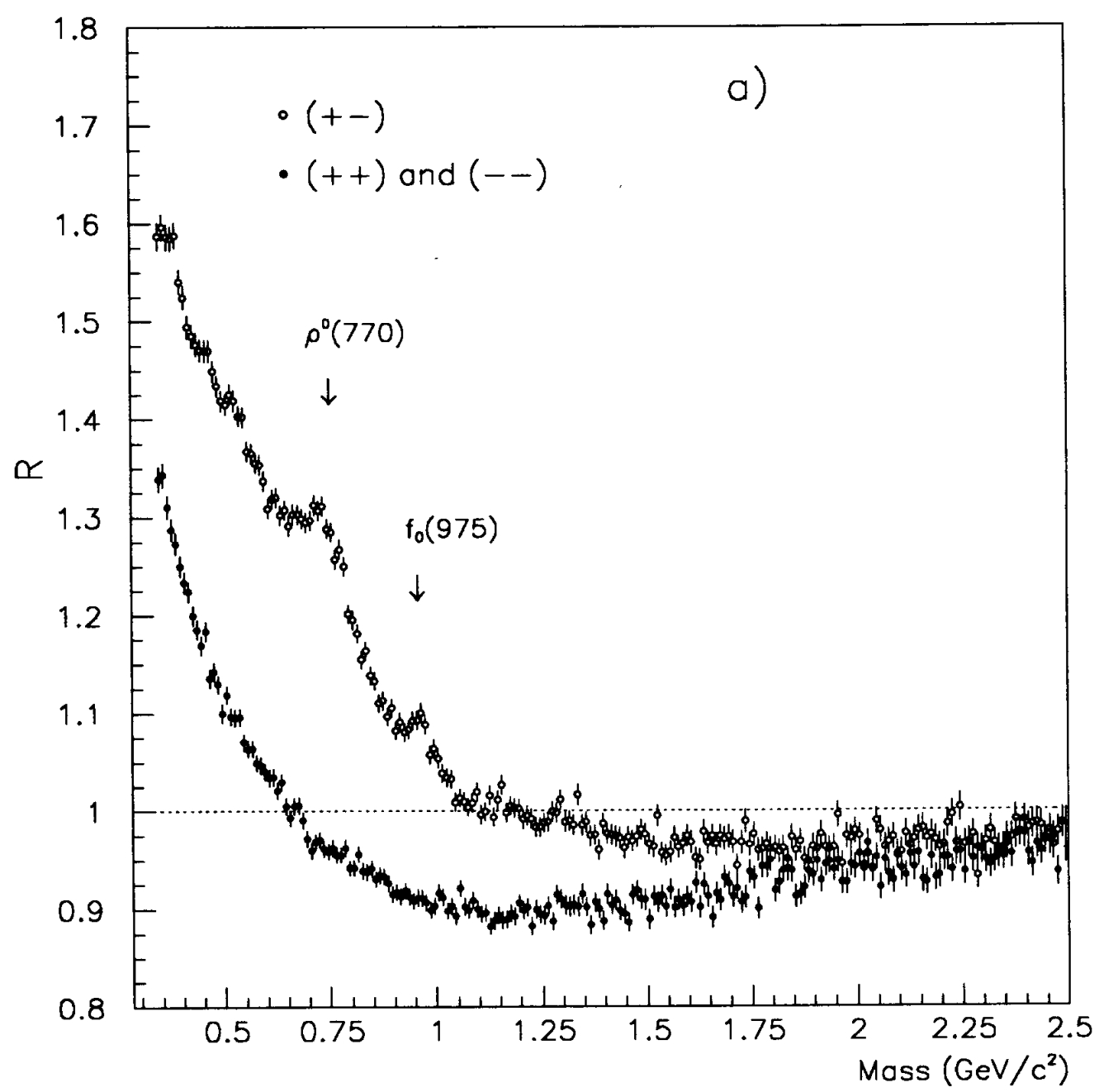

Fig.3a 


\section{DELPHI}

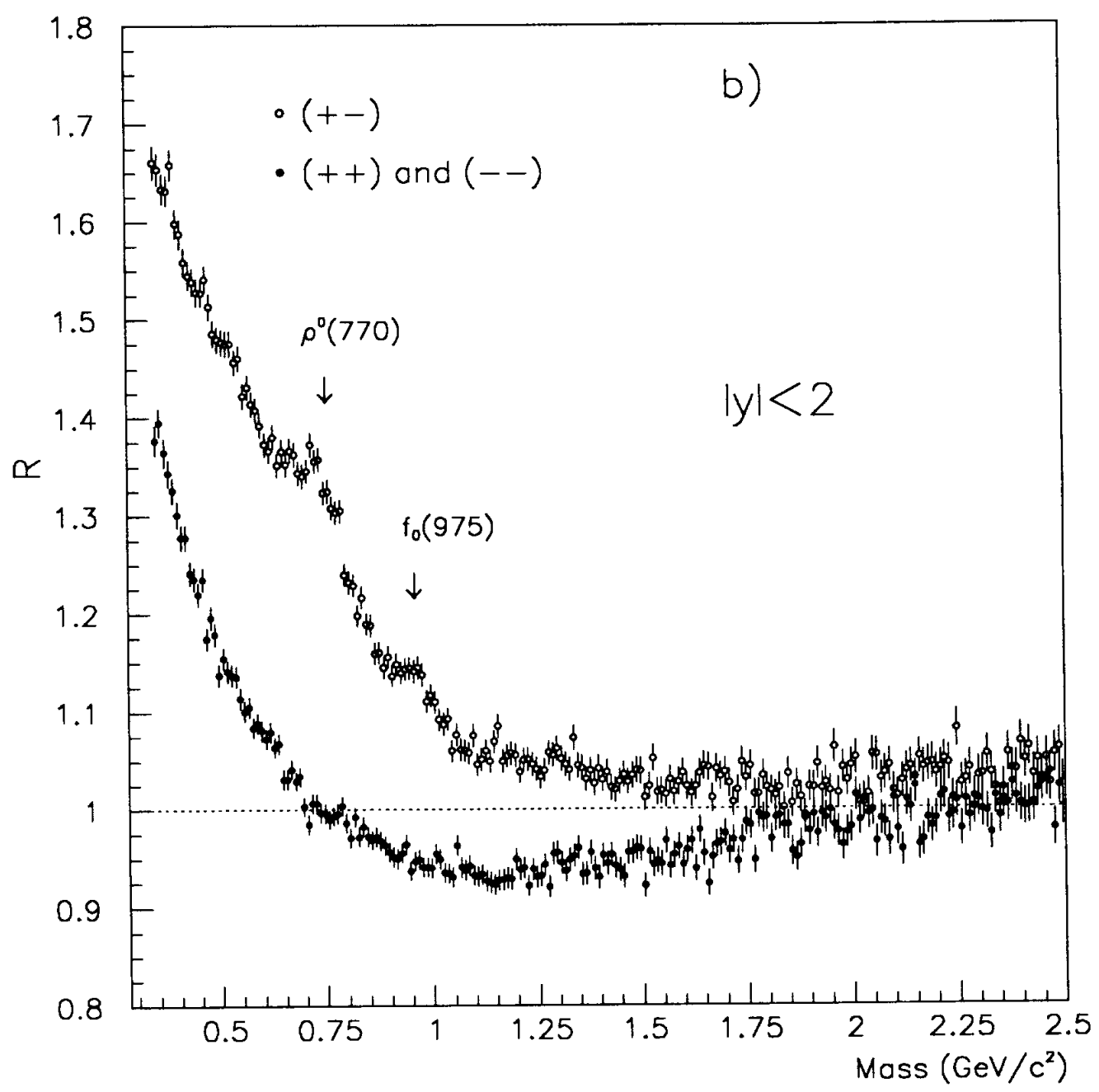

Fig.3b 

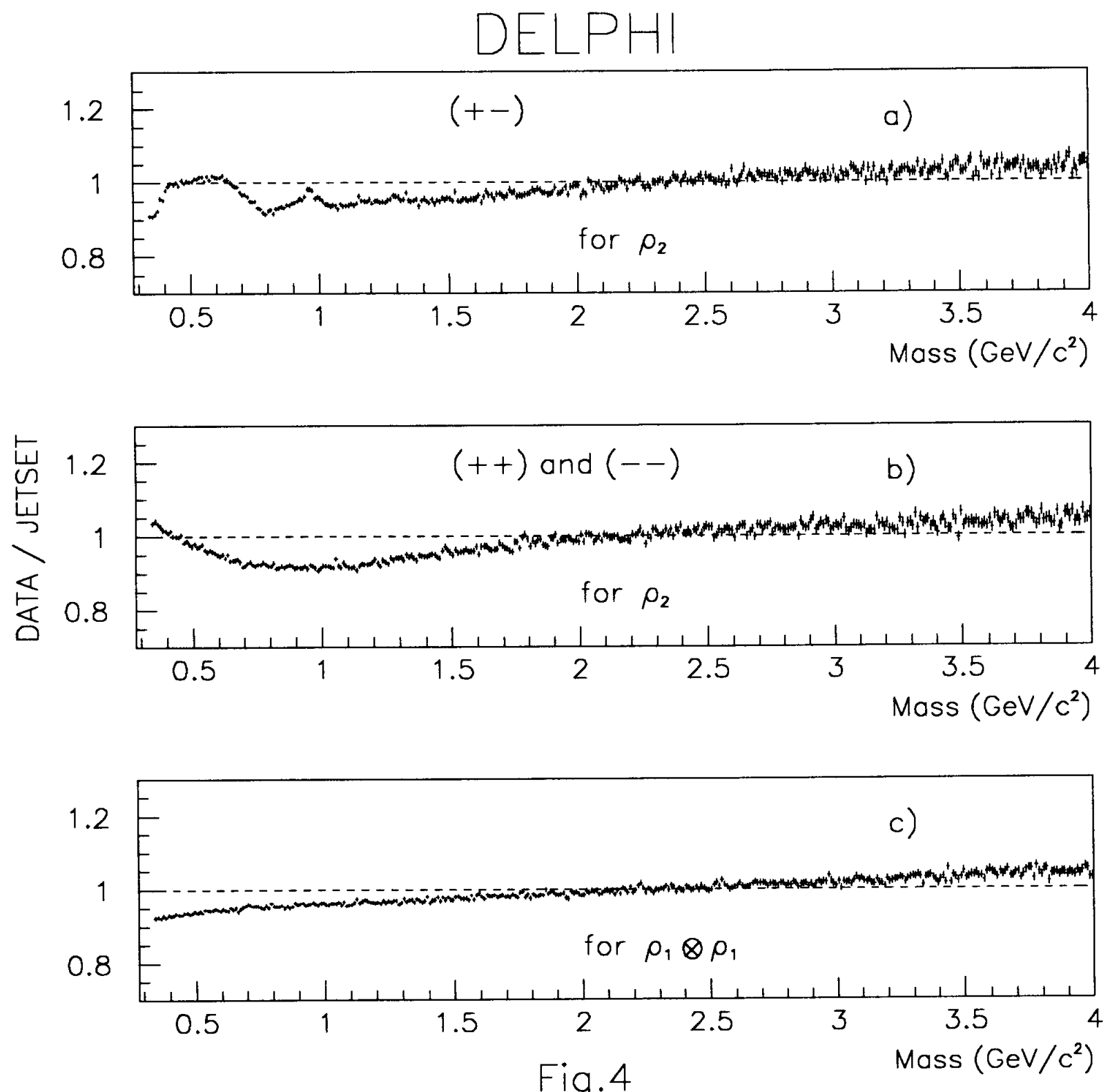


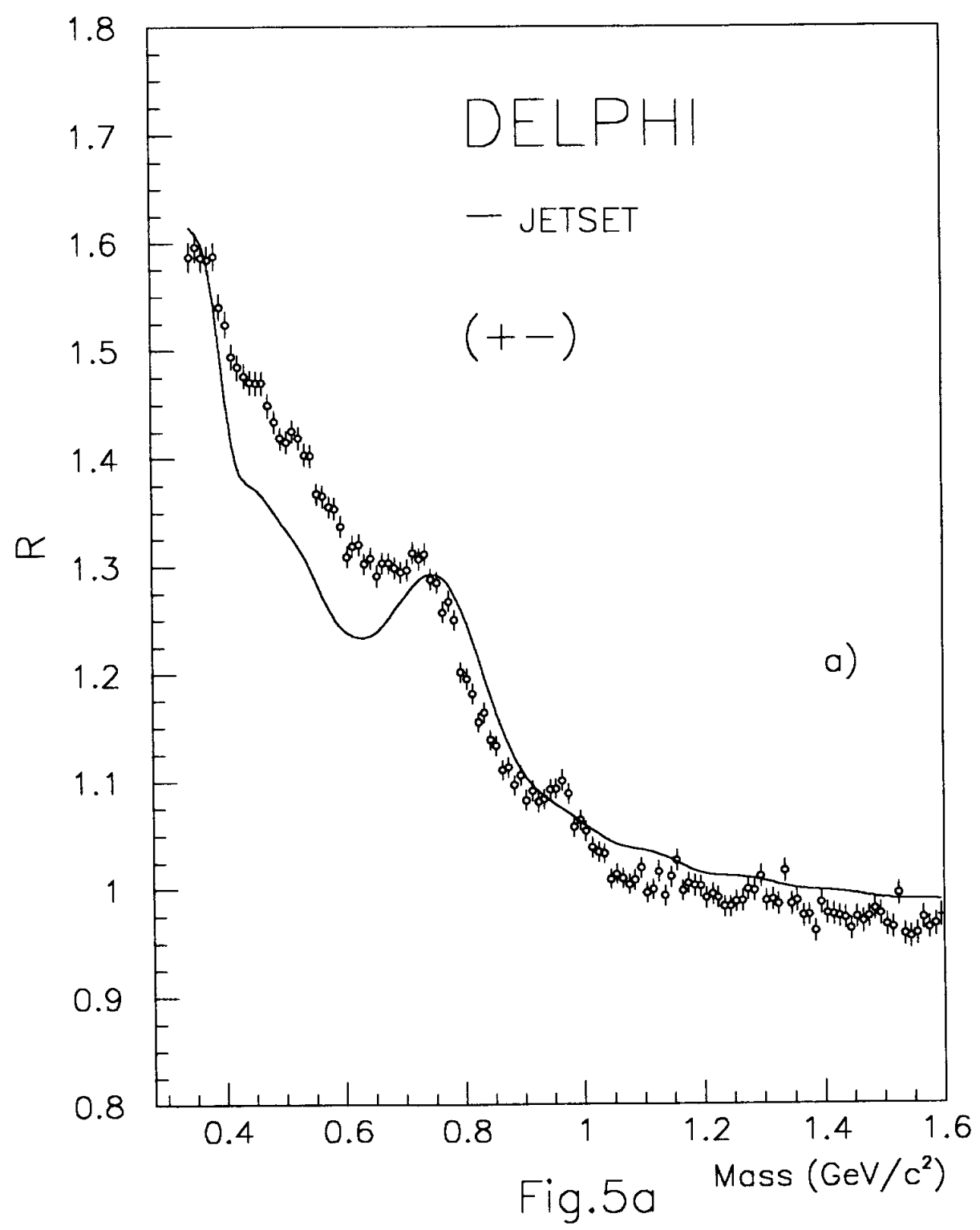




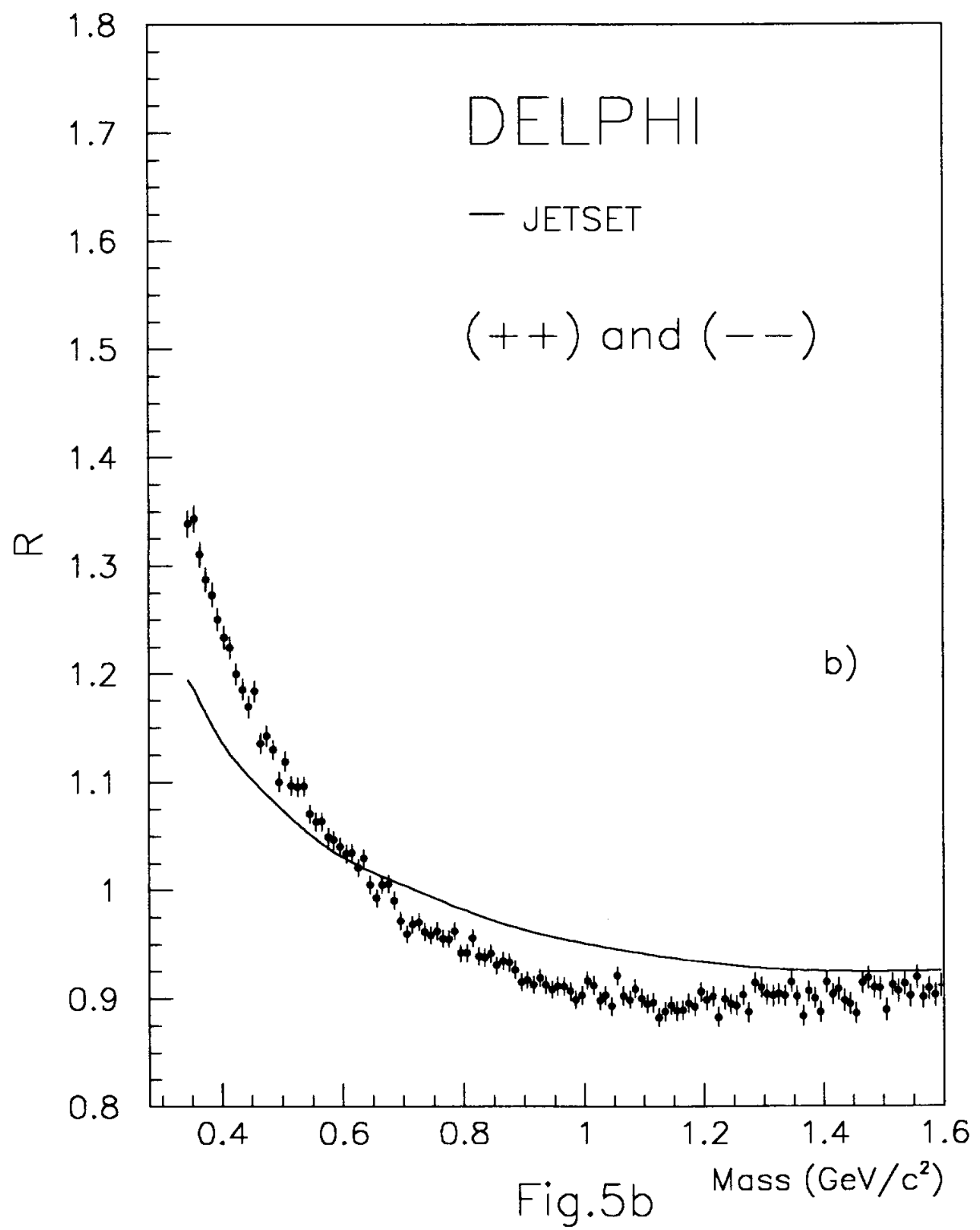




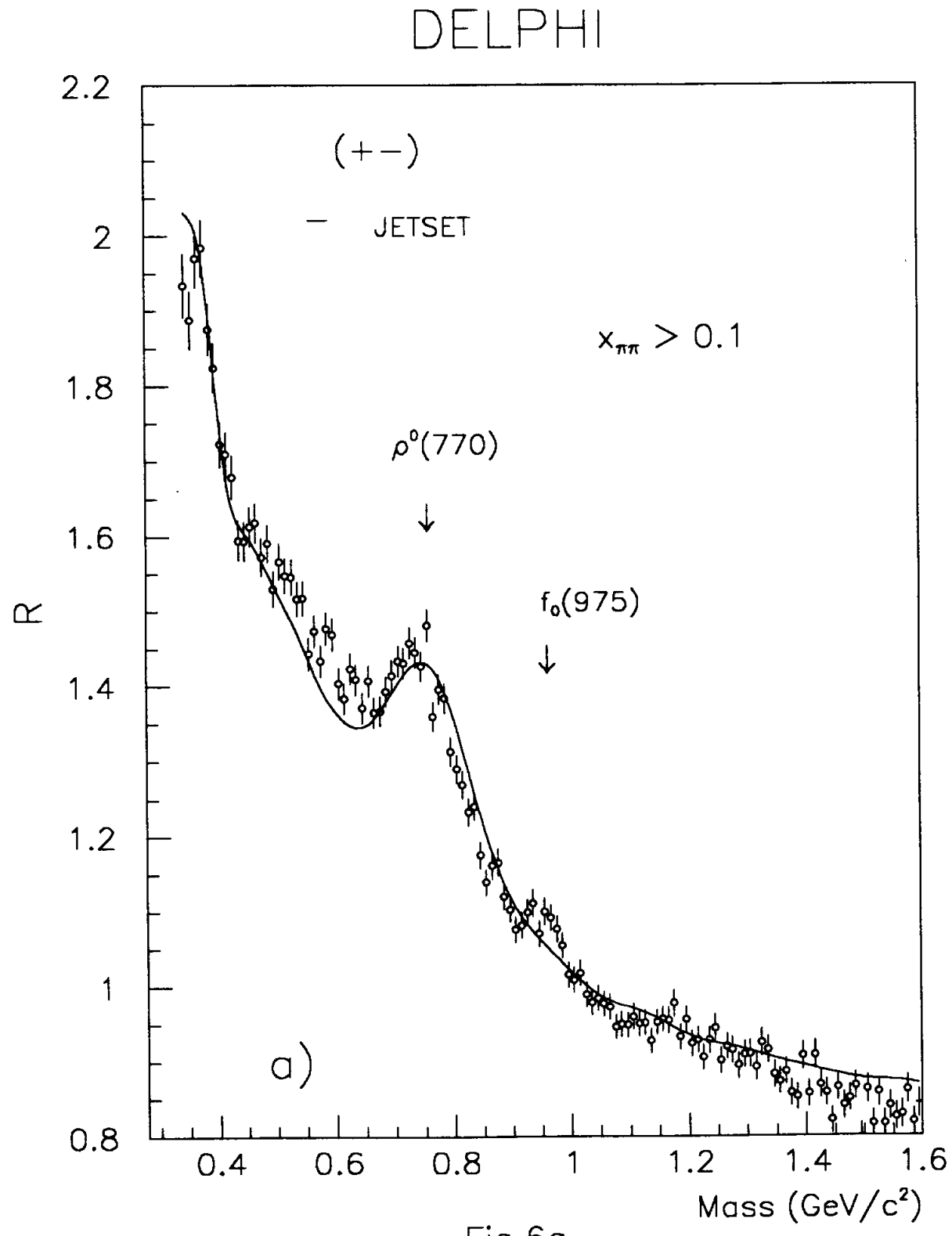

Fig.6o 


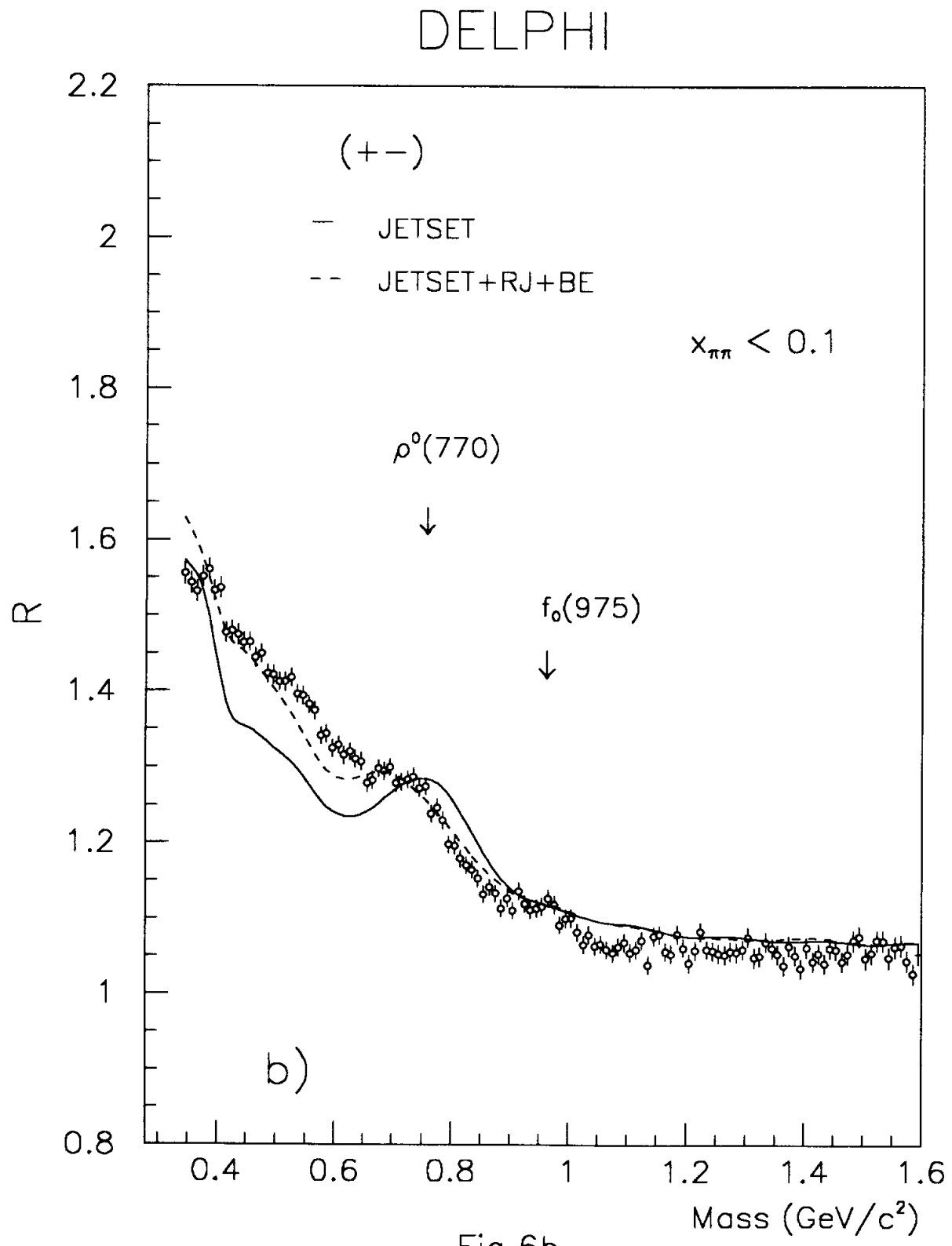

Fig.6b 
DELPHI

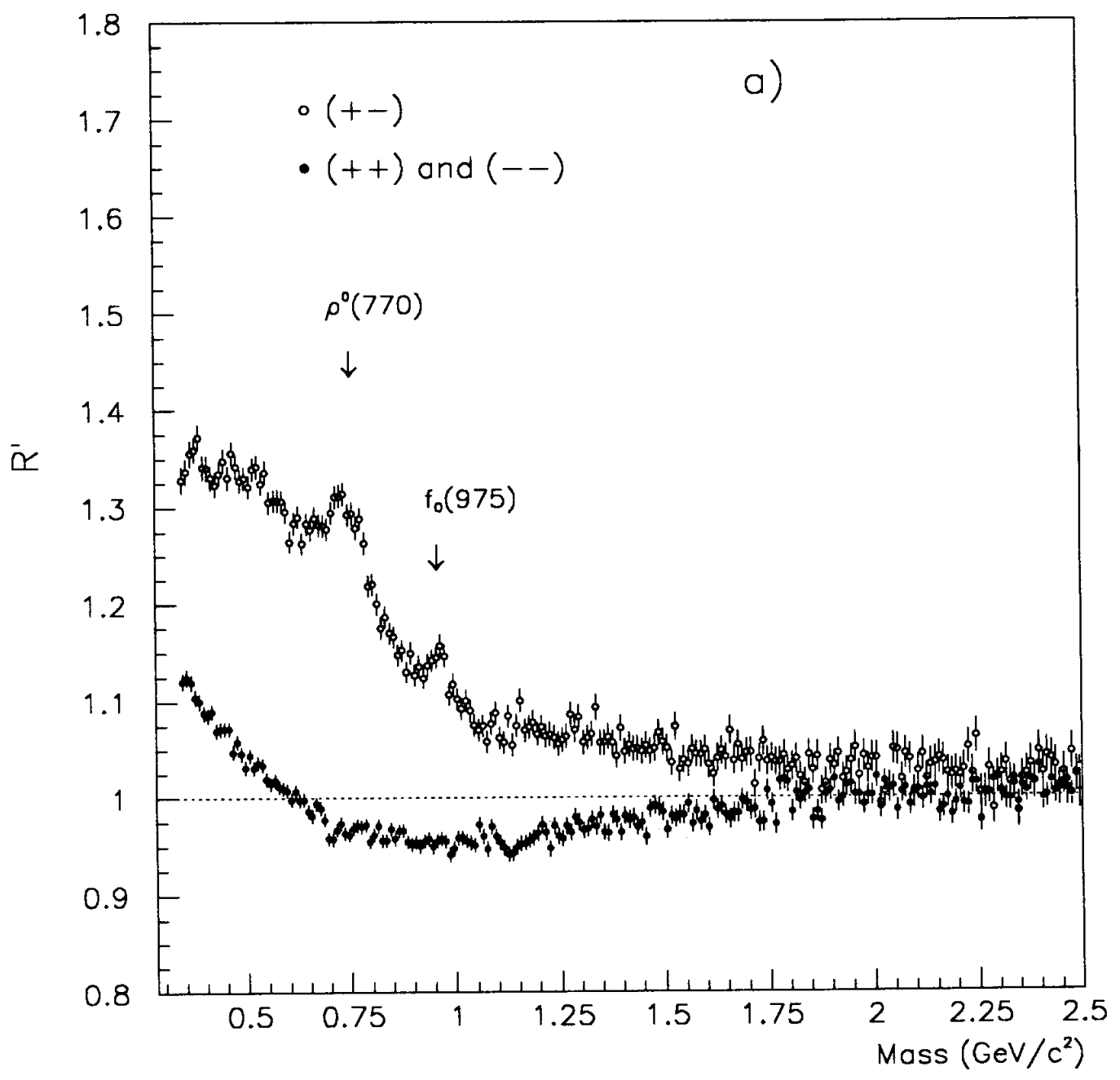

Fig.7o 


\section{DELPHI}

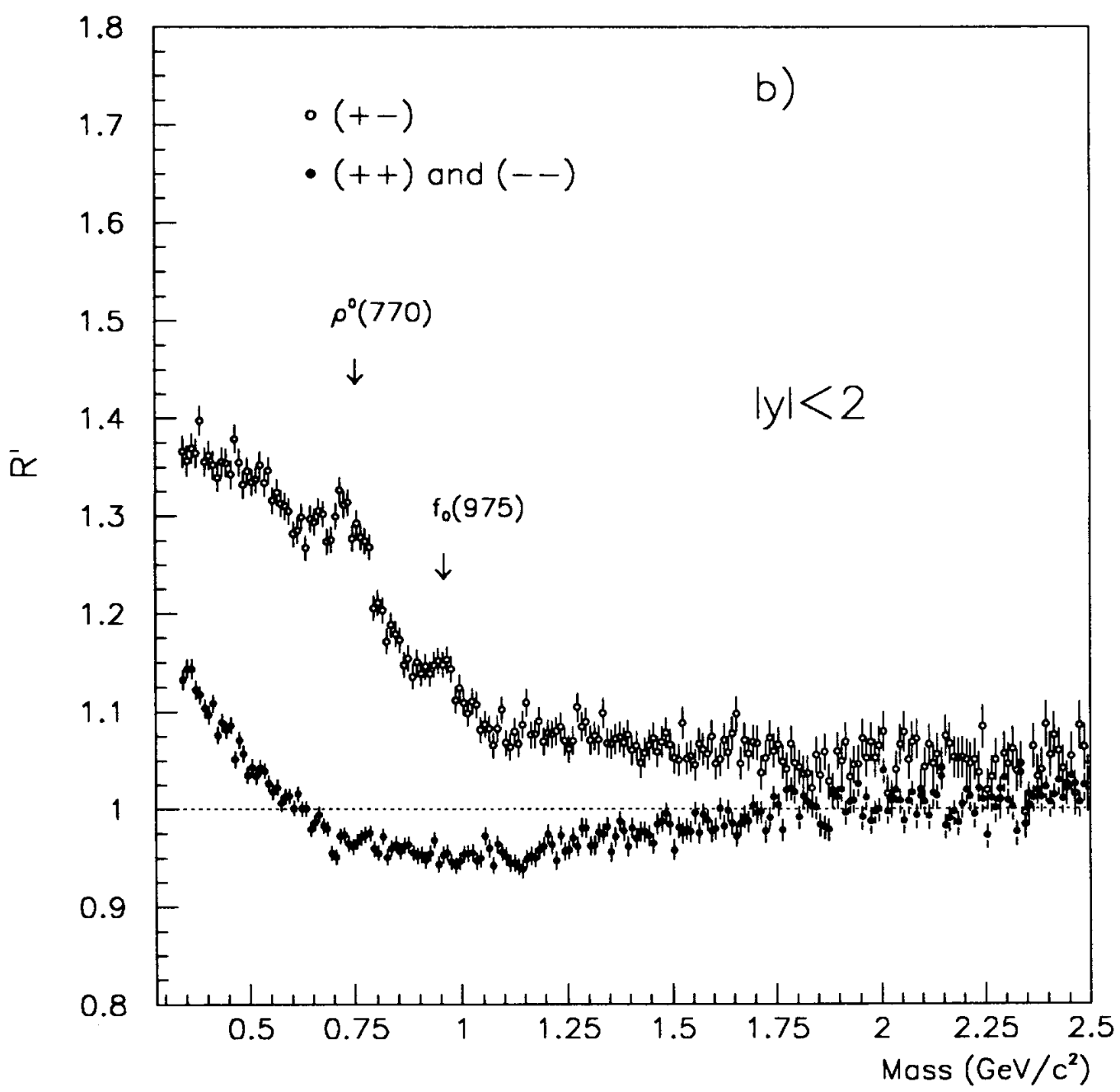

Fig. 7b 


\section{DELPHI}
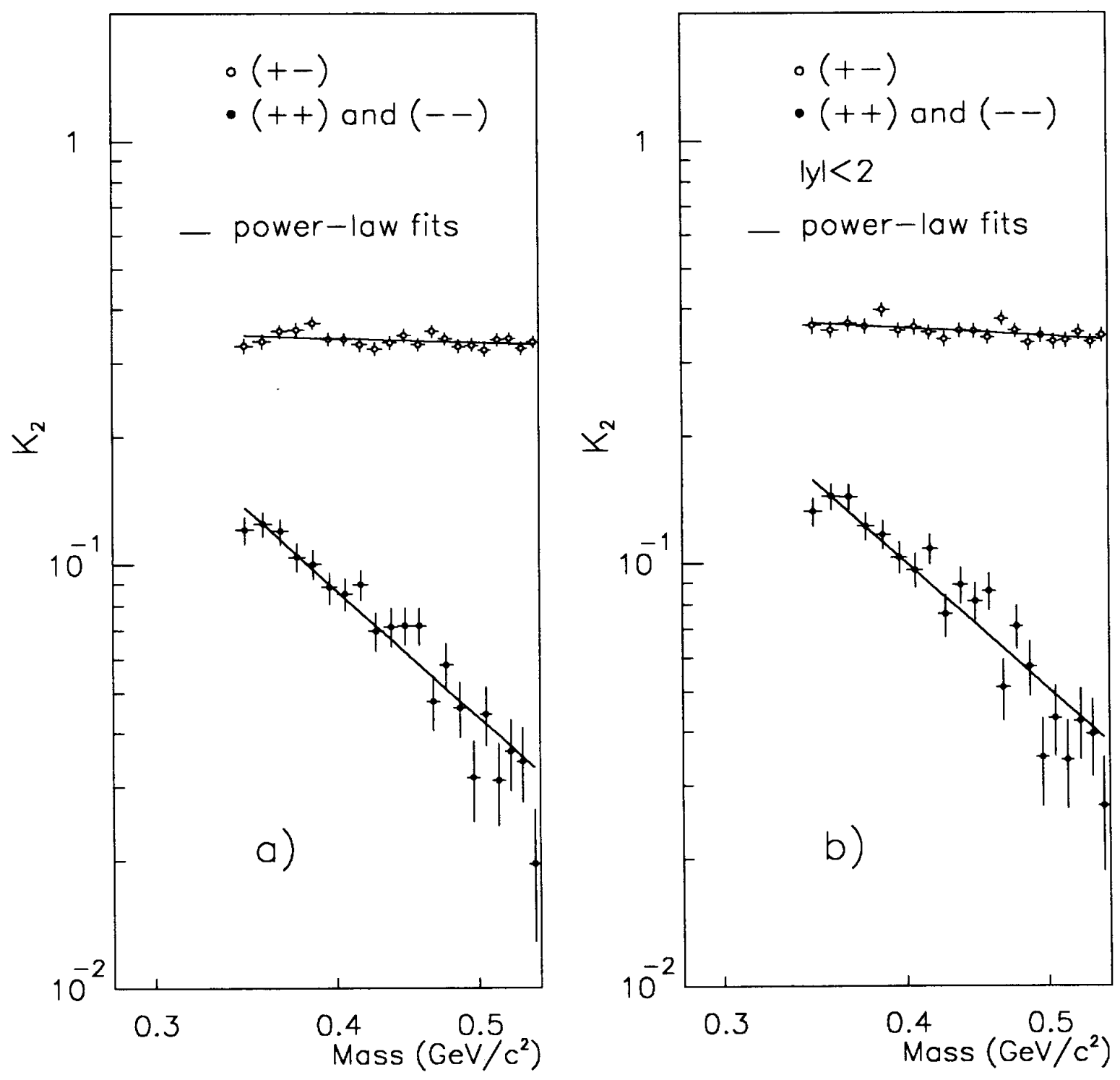

Fig. 8 


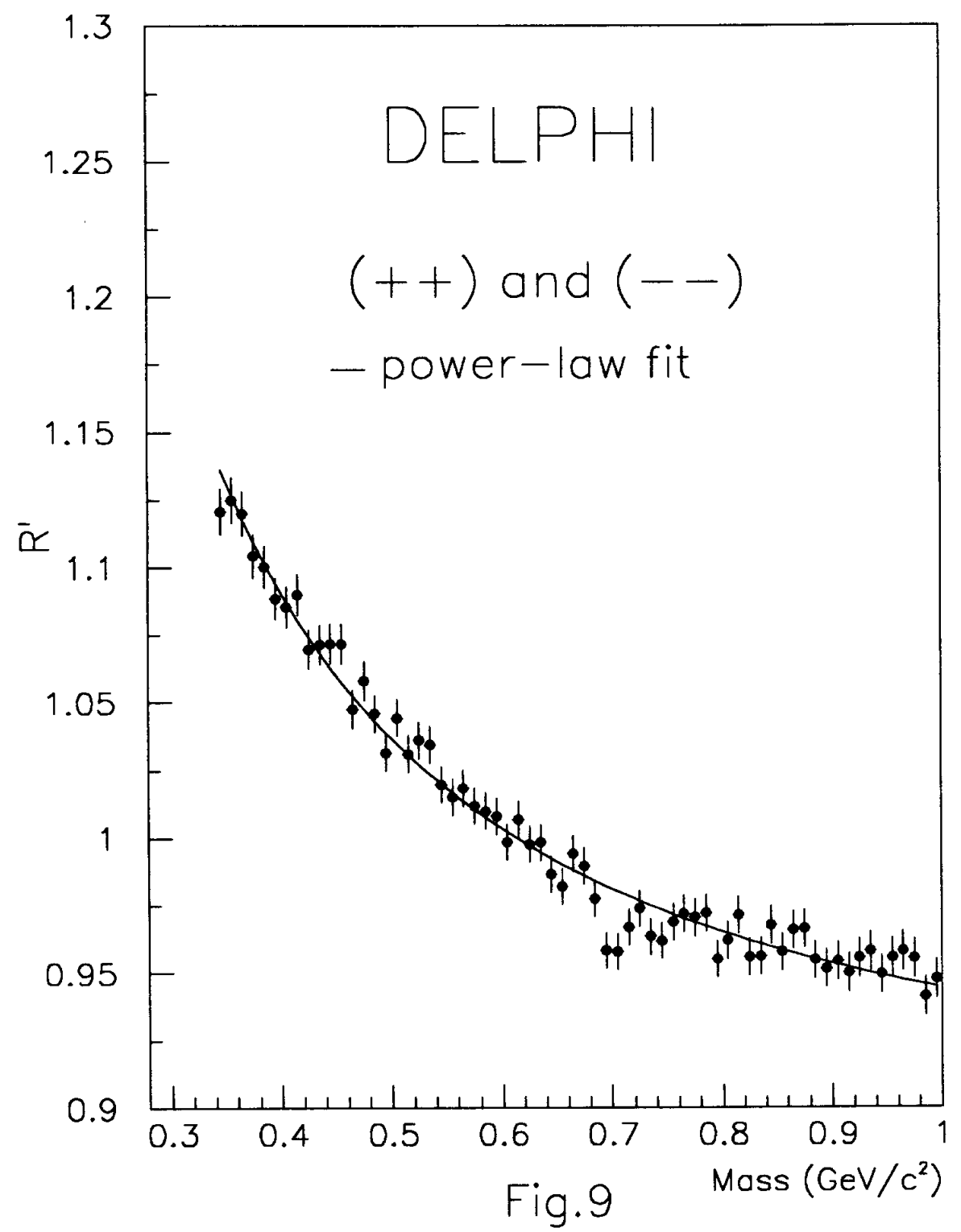



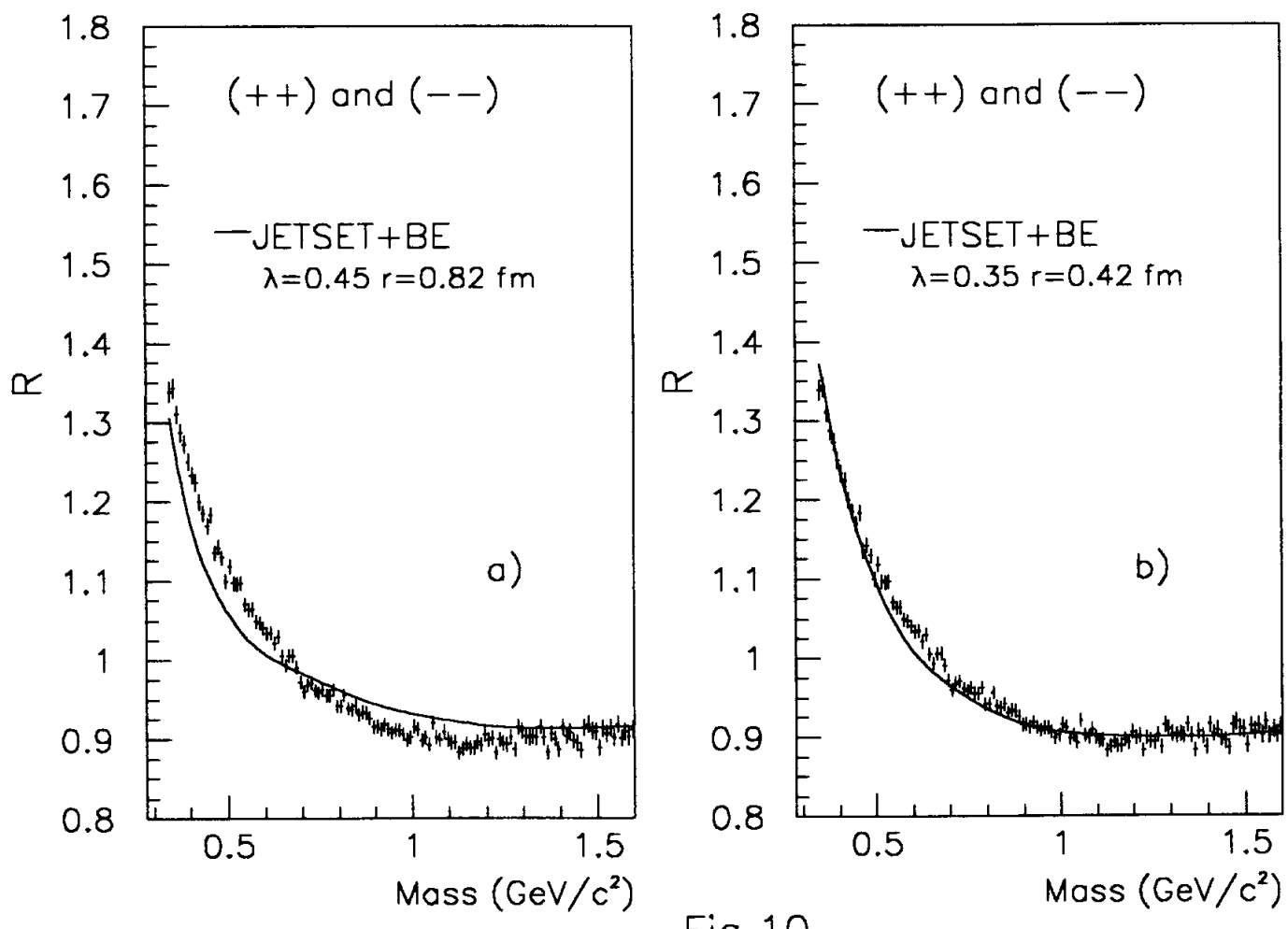

Fig. 10 


\section{DELPHI}
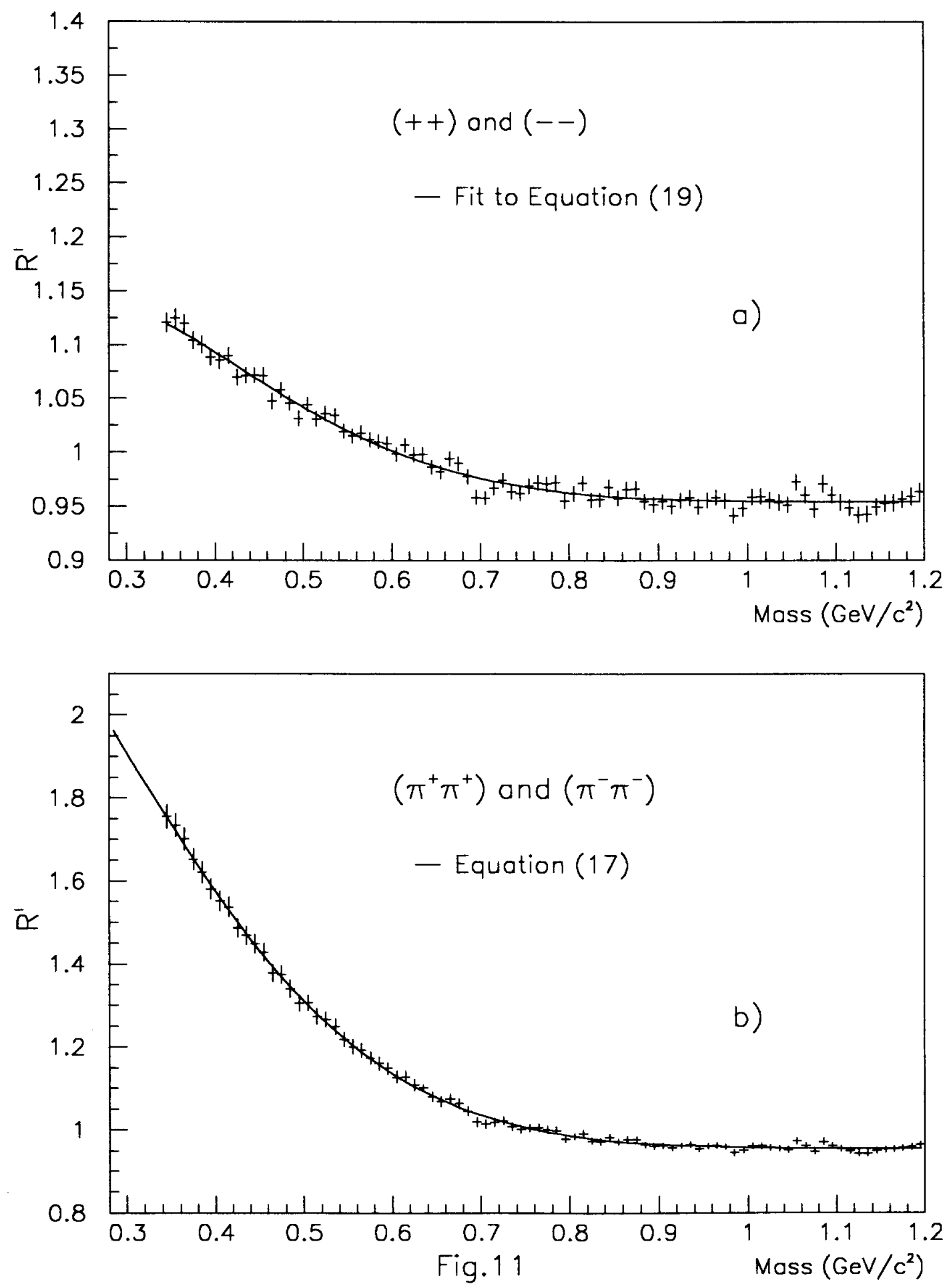


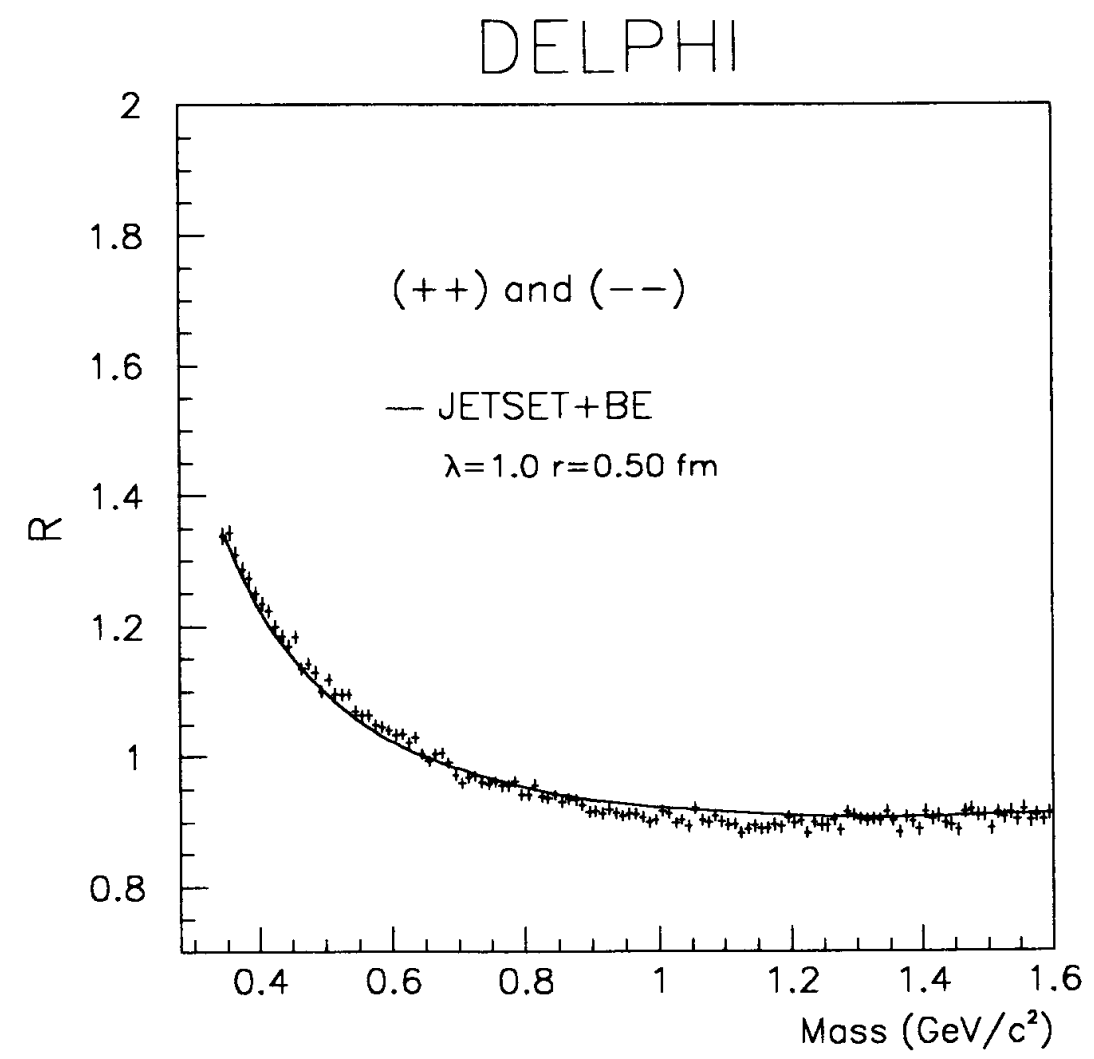

Fig. 12 


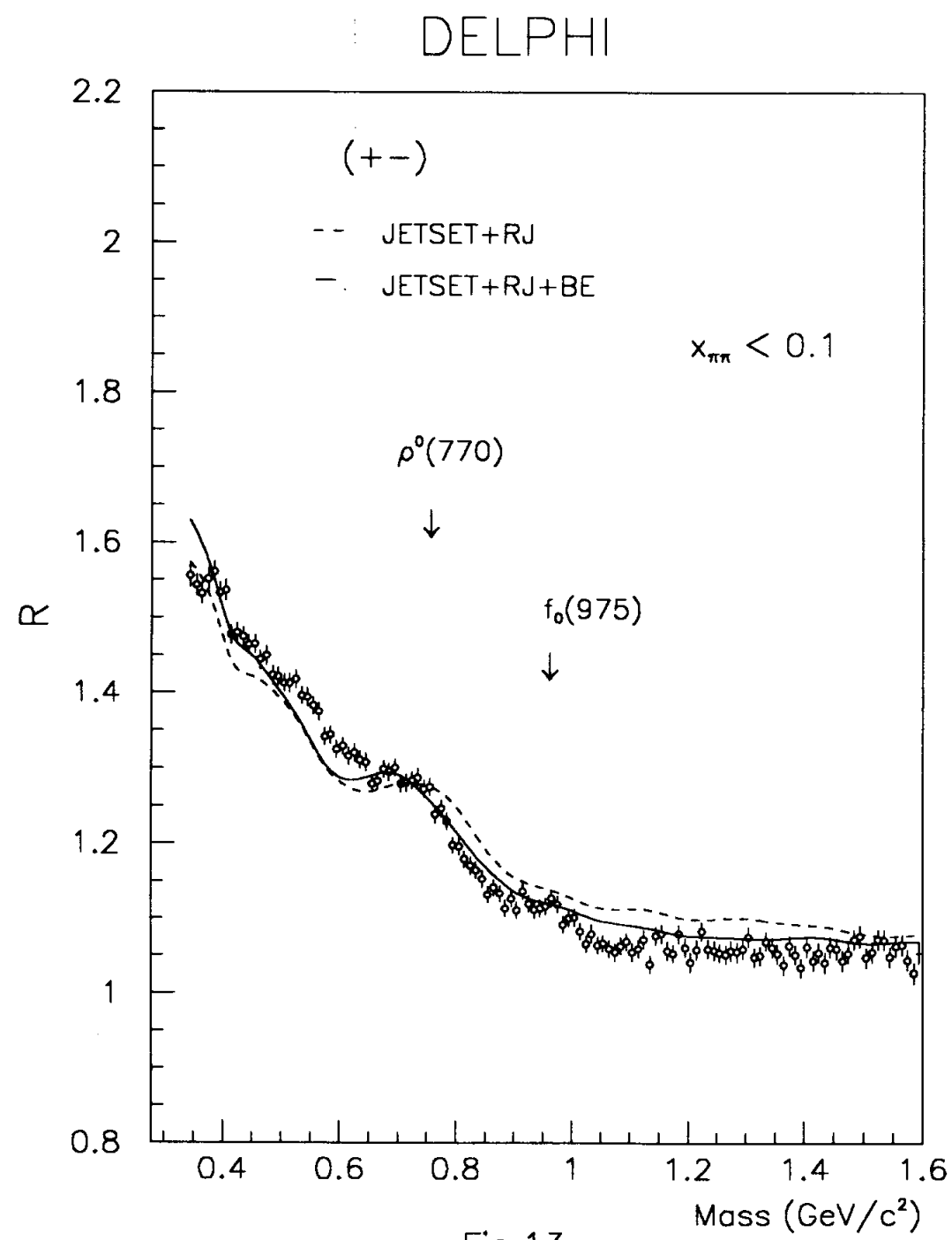

Fig.13 